\title{
Power Transport Theorem Based Decoupling Mode Theory for Wave-guiding Structures
}

\author{
Ren-Zun Lian and Ming-Yao Xia, Senior Member, IEEE
}

\begin{abstract}
Power transport theorem (PTT) governing the transport process of the power-flow passing through waveguide is derived. The input power as the source term in PTT is found to be the one for sustaining a stationary power transport, and the corresponding input power operator (IPO) is formulated. The travelling-wave condition satisfied by the travelling-wave modes one-directionally propagating along the waveguide is introduced as a counterpart of the famous Sommerfeld's radiation condition satisfied by the radiative fields distributing in far zone. Employing the travelling-wave condition and some other necessary conditions, the dependent currents in IPO are effectively eliminated. Under PTT framework, the recently developed decoupling mode theory (DMT) for wave-port-fed antennas is further generalized to waveguides. The PTT-based DMT (PTT-DMT) focuses on constructing a set of energy-decoupled modes (DMs) for any pre-selected objective waveguide by orthogonalizing the IPO with only independent current, and any two different DMs don't have net energy exchange in an integral period. The PTT-DMT is an effective alternative for classical eigen-mode theory. The alternative realizes an effective unification for waveguide-oriented modal analysis theory and antenna-oriented modal analysis theory, such that the theories can be easily integrated into a single theory for whole waveguide-antenna combined system in the future.
\end{abstract}

Index Terms-Decoupling mode theory (DMT), energy-decoupled mode (DM), input power operator (IPO), power transport theorem (PTT), travelling-wave condition, waveguide.

\section{INTRODUCTION}

$\mathrm{W}$ AVE-GUIDING structure (i.e., waveguide) is the one for guiding the propagation of electromagnetic (EM) energy in a well-defined direction [1]-[2], and it has become a kind of important transmission lines applied in many practical radio-frequency systems [3]-[5]. Waveguide usually comprises a hollow conducting tube within which EM energies may be propagated or a solid dielectric or dielectric-filled conductor for the same purpose [1]. For purposes of definition and description relating to wave propagation, waveguides are usually assumed to be of invariant cross section along the direction of propagation [1]. A typical hollow metallic waveguide, which is used to guide EM energy from signal generator to transmitting antenna, is shown in Fig. 1.

Manuscript with ID [TMTT-2021-04-0464] was submitted to IEEE-TMTT on April 14, 2021. (Corresponding authors: Ren-Zun Lian; Ming-Yao Xia).

R. Z. Lian and M. Y. Xia are with the Department of Electronics, School of Electronics Engineering and Computer Science, Peking University, Beijing 100871, China. (e-mail: rzlian@vip.163.com; myxia@pku.edu.cn).

Manuscript ID: TMTT-2021-04-0464.

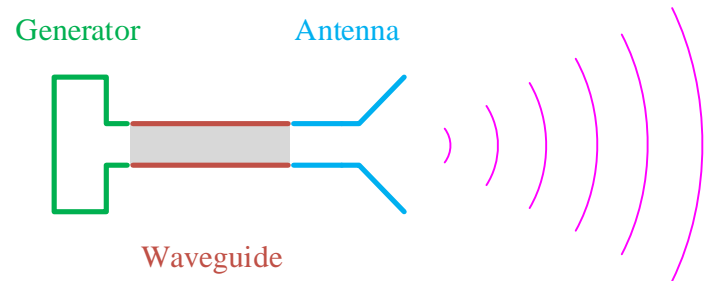

Fig. 1. Geometry of a typical transmitting system constituted by signal generator, metallic tube waveguide, and metallic horn antenna.

During the working process of whole waveguide-antenna system, there exists a strong inter-action between the EM fields generated by waveguide and antenna [6]-[8]. The inter-action will lead to a complicated inter-transformation/inter-excitation between the fundamental modes of waveguide and the fundamental modes of antenna [9]-[10]. Modal matching method [10]-[15] is an effective one for quantitatively describing and analyzing the modal inter-transformation/inter-excitation. However, before utilizing the modal matching method, it is indispensable to separately calculate the fundamental modes of waveguide and the fundamental modes of antenna (i.e., to do the modal analysis for waveguide and the modal analysis for antenna separately) beforehand.

For waveguides, Sturm-Liouville eigen-mode theory (EMT) [16]-[19] is one of the most frequently used modal analysis methods. By solving the eigenvalue equation formulated by Sturm-Liouville operator, the EMT can construct a set of energy-decoupled fundamental working modes for waveguides [2]-[3]. In EM engineering, there have existed some different computational methods for solving the eigenvalue equation, such as analytical method [20]-[23], variational method [24]-[26], approximate method [27]-[29], numerical method (e.g. finite element method [30]-[34], finite difference method [35], and method of moments [36]), and semi-analytical semi-numerical method [37]-[38].

For antennas, the earliest modal analysis methods are some EMT-based modal expansion methods [39]-[41] and some model-based approximate methods (e.g. cavity model method [42] and dielectric waveguide model method [43]). For the last several years, the characteristic mode theory (CMT), which was established by Garbacz [44]-[46] and Harrington [47]-[50] et al., are frequently employed to analyze some antennas [51]-[53]. Very recently, it is found out that [54]: strictly speaking, the CMT is a modal analysis method for scatterers rather than for antennas, so the existing antenna-oriented characteristic mode analysis are approximate but not rigorous. 
In [54], a rigorous antenna-oriented modal analysis method — decoupling mode theory (DMT) — is established under power transport theorem (PTT) framework. By orthogonalizing input power operator (IPO), the DMT can construct a set of energy-decoupled fundamental working modes for wave-port-fed transmitting antennas. Both the waveguide-oriented EMT and the antenna-oriented DMT are rigorous modal analysis theories, but it is inconvenient to integrate them into together, because the modal generating operator used in the former is a two-order differential operator Sturm-Liouville operator - but the modal generating operator used in the latter is a power-type integral operator - IPO.

To resolve this problem, this paper is devoted to further generalizing the antenna-oriented DMT to waveguides, such that the modal analysis for waveguides and antennas can be done under a unified framework - PTT, and the unification will become the theoretical support to do the modal analysis for the whole waveguide-antenna combined system in the future studies. This paper is organized as follows: Sec. II establishes the PTT-based DMT (PTT-DMT) for metallic waveguides; Sec. III generalizes the idea of Sec. II to material waveguides; Sec. IV concludes this paper; the appendices provide some detailed mathematical formulations related to this paper. The $e^{j \omega t}$ convention and inner product $<\boldsymbol{f}, \boldsymbol{g}>_{\Omega}=\int_{\Omega} \boldsymbol{f}^{\dagger} \cdot \boldsymbol{g} d \Omega$ are used throughout, where " $\dagger$ " is conjugate transpose operation.

\section{PTT-DMT For Metallic WaVeguides}

In this section, the metallic waveguide shown in Fig. 2 is considered, and its longitudinal direction is selected as Z-axis, and its cross section can be arbitrary geometry. In the figure, only the part sandwiched between two cross sections $S^{\mathrm{i}}$ and $S^{\circ}$ is illustrated, and the longitudinal length of the part is $L$. The waveguide wall of the part is denoted as $S^{\mathrm{g}}$. The waveguide cavity (i.e., the region enclosed by the closed surface $\left.S^{\mathrm{i}} \cup S^{\mathrm{g}} \cup S^{\mathrm{o}}\right)$ is denoted as $V$, and the cavity is filled by the material with parameters $(\mu, \varepsilon)$.

\section{A. Power Transport Theorem (PTT) and Input Power Oper- ator (IPO)}

The EM fields $(\boldsymbol{E}, \boldsymbol{H})$ distributing on region $V$ satisfy Maxwell's equations $\nabla \times \boldsymbol{H}=j \omega \varepsilon \boldsymbol{E}$ and $\nabla \times \boldsymbol{E}=-j \omega \mu \boldsymbol{H}$, and the equations imply the following power relation

$$
P^{\text {in }}=P^{\text {out }}+j 2 \omega\left(W^{\mathrm{mag}}-W^{\text {ele }}\right)
$$

Here, $P^{\text {out }}=(1 / 2) \iint_{\mathrm{S}^{\mathrm{o}}}\left(\boldsymbol{E} \times \boldsymbol{H}^{\dagger}\right) \cdot d \boldsymbol{S}$ and $W^{\mathrm{mag}}=(1 / 4)<\boldsymbol{H}, \boldsymbol{B}>_{V}$ and $W^{\text {ele }}=(1 / 4)<\grave{D}, \boldsymbol{E}>_{V}$, and

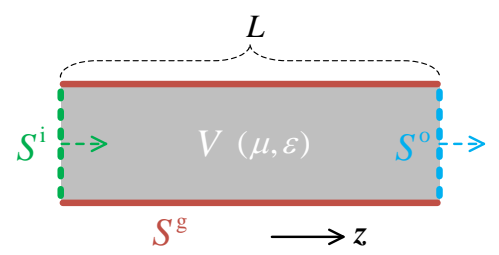

Fig. 2. Topological structure of a metallic tube waveguide section with longitudinal length $L$.

$$
P^{\text {in }}=(1 / 2) \iint_{S^{i}}\left(\boldsymbol{E} \times \boldsymbol{H}^{\dagger}\right) \cdot d \boldsymbol{S}
$$

where the normal reference directions of $S^{\mathrm{i}}$ and $S^{\mathrm{o}}$ are Z-axis as shown in Fig. 2.

Power relation (1) is a quantitative depiction for the transport process of the power-flow passing through region $V$. Specifically, power $P^{\text {in }}$, which passes through $S^{\mathrm{i}}$, is the power inputted into $V$, and it is finally transformed into the following two parts:

Part 1. the power $P^{\text {out }}$ outputted from $V$ by passing through $S^{\circ}$ and

Part 2. the power $2 \omega\left(W^{\text {mag }}-W^{\text {ele }}\right)$ used to contribute the energy stored in $V$.

Following the convention used in [54], power relation (1) is called power transport theorem (PTT) in this paper, and it will be selected as the framework used to establish the decoupling mode theory (DMT) for metallic waveguides in this section. In addition, based on the above these, the port $S^{\mathrm{i}} / S^{\mathrm{o}}$ and power $P^{\text {in }} / P^{\text {out }}$ are called input/output port and input/output power respectively, and the input power operator (IPO) will be employed to calculate the energy-decoupled modes (DMs) of the waveguide.

In (2), the IPO is expressed as a field-field interaction. In fact, if the equivalent electric and magnetic currents distributing on $S^{\mathrm{i}}$ are denoted as $\boldsymbol{J}^{\mathrm{i}}$ and $\boldsymbol{M}^{\mathrm{i}}$, which are defined as $\boldsymbol{J}^{\mathrm{i}}=\boldsymbol{z} \times \boldsymbol{H}$ and $\boldsymbol{M}^{\mathrm{i}}=\boldsymbol{E} \times \boldsymbol{z}$, then the IPO can be alternatively expressed as field-current interactions $\left.P^{\text {in }}=-(1 / 2)<\boldsymbol{J}^{\mathrm{i}}, \boldsymbol{E}\right\rangle_{S^{\mathrm{i}}}$ and $P^{\text {in }}=-(1 / 2)<\boldsymbol{H}, \boldsymbol{M}^{\mathrm{i}}>_{S^{\mathrm{i}}}$. To effectively distinguish the three different expressions from each other, the expressions are respectively called EH, JE, and HM interaction forms.

If the equivalent currents distributing on $S^{\circ}$ and the induced electric current distributing on $S^{\mathrm{g}}$ are denoted as $\left(\boldsymbol{J}^{\mathrm{o}}, \boldsymbol{M}^{\mathrm{o}}\right)$ and $\boldsymbol{J}^{\mathrm{g}}$ respectively, where $\boldsymbol{J}^{\mathrm{o}}$ and $\boldsymbol{M}^{\mathrm{o}}$ are defined as $\boldsymbol{J}^{\mathrm{o}}=\boldsymbol{z} \times \boldsymbol{H}$ and $\boldsymbol{M}^{\mathrm{o}}=\boldsymbol{E} \times \boldsymbol{z}$ respectively, then the JE and HM interaction forms of IPO are immediately rewritten as follows:

$$
\begin{aligned}
P^{\text {in }} & =-(1 / 2)\left\langle\boldsymbol{J}^{\mathrm{i}}, \mathcal{E}\left(\boldsymbol{J}^{\mathrm{i}}+\boldsymbol{J}^{\mathrm{g}}-\boldsymbol{J}^{\mathrm{o}}, \boldsymbol{M}^{\mathrm{i}}-\boldsymbol{M}^{\mathrm{o}}\right)\right\rangle_{S_{+}^{\mathrm{i}}} \\
& =-(1 / 2)\left\langle\boldsymbol{M}^{\mathrm{i}}, \mathcal{H}\left(\boldsymbol{J}^{\mathrm{i}}+\boldsymbol{J}^{\mathrm{g}}-\boldsymbol{J}^{\mathrm{o}}, \boldsymbol{M}^{\mathrm{i}}-\boldsymbol{M}^{\mathrm{o}}\right)\right\rangle_{S_{+}^{\mathrm{i}}}^{\dagger}
\end{aligned}
$$

where the operators are $\mathcal{E}(\boldsymbol{J}, \boldsymbol{M})=-j \omega \mu \mathcal{L}(\boldsymbol{J})-\mathcal{K}(\boldsymbol{M})$ and $\mathcal{H}(\boldsymbol{J}, \boldsymbol{M})=\mathcal{K}(\boldsymbol{J})-j \omega \varepsilon \mathcal{L}(\boldsymbol{M})$, and the integral surface $S_{+}^{\mathrm{i}}$ is the right-side surface of $S^{\mathrm{i}}$.

Employing the basis function expansions for the currents, the JE and HM forms of IPO are discretized into the following matrix forms

$$
P^{\text {in }}=\left(\mathrm{J}^{\mathrm{i}}\right)^{\dagger} \cdot \mathrm{P}_{\mathrm{JE}}^{\text {in }} \cdot\left[\begin{array}{l}
\mathrm{J}^{\mathrm{i}} \\
\mathrm{J}^{\mathrm{g}} \\
\mathrm{J}^{\mathrm{o}} \\
\mathrm{M}^{\mathrm{i}} \\
\mathrm{M}^{\mathrm{o}}
\end{array}\right]=\left[\begin{array}{l}
\mathrm{J}^{\mathrm{i}} \\
\mathrm{J}^{\mathrm{g}} \\
\mathrm{J}^{\mathrm{o}} \\
\mathrm{M}^{\mathrm{i}} \\
\mathrm{M}^{\mathrm{o}}
\end{array}\right]^{\dagger} \cdot \mathrm{P}_{\mathrm{HM}}^{\text {in }} \cdot \mathrm{M}^{\mathrm{i}}
$$

where $\mathrm{J}^{\mathrm{i} / g / \mathrm{o}}$ and $\mathrm{M}^{\mathrm{i} / \mathrm{o}}$ are the expansion coefficient vectors for $\boldsymbol{J}^{\mathrm{i} / \mathrm{g} / \mathrm{o}}$ and $\boldsymbol{M}^{\mathrm{i} / \mathrm{o}}$ respectively. 


\section{B. Dependent Variable Elimination (DVE)}

The currents involved in the IPO are not independent, because they satisfy the following integral equations

$$
\begin{aligned}
& {\left[\mathcal{H}\left(\boldsymbol{J}^{\mathrm{i}}+\boldsymbol{J}^{\mathrm{g}}-\boldsymbol{J}^{\mathrm{o}}, \boldsymbol{M}^{\mathrm{i}}-\boldsymbol{M}^{\mathrm{o}}\right)\right]_{S_{+}^{\mathrm{i}}}^{\mathrm{tan}}=\boldsymbol{J}^{\mathrm{i}} \times \boldsymbol{z}} \\
& {\left[\mathcal{E}\left(\boldsymbol{J}^{\mathrm{i}}+\boldsymbol{J}^{\mathrm{g}}-\boldsymbol{J}^{\mathrm{o}}, \boldsymbol{M}^{\mathrm{i}}-\boldsymbol{M}^{\mathrm{o}}\right)\right]_{S_{+}^{\mathrm{i}}}^{\mathrm{tan}}=\boldsymbol{z} \times \boldsymbol{M}^{\mathrm{i}}} \\
& {\left[\mathcal{E}\left(\boldsymbol{J}^{\mathrm{i}}+\boldsymbol{J}^{\mathrm{g}}-\boldsymbol{J}^{\mathrm{o}}, \boldsymbol{M}^{\mathrm{i}}-\boldsymbol{M}^{\mathrm{o}}\right)\right]_{S^{\mathrm{g}}}^{\text {tan }}=0} \\
& {\left[\mathcal{E}\left(\boldsymbol{J}^{\mathrm{i}}+\boldsymbol{J}^{\mathrm{g}}-\boldsymbol{J}^{\mathrm{o}}, \boldsymbol{M}^{\mathrm{i}}-\boldsymbol{M}^{\mathrm{o}}\right)\right]_{S_{-}^{\mathrm{o}}}^{\text {tan }}=\left[\mathcal{E}\left(\boldsymbol{J}^{\mathrm{o}}, \boldsymbol{M}^{\mathrm{o}}\right)\right]_{S_{+}^{\mathrm{o}}}^{\text {tan }}} \\
& {\left[\mathcal{H}\left(\boldsymbol{J}^{\mathrm{i}}+\boldsymbol{J}^{\mathrm{g}}-\boldsymbol{J}^{\mathrm{o}}, \boldsymbol{M}^{\mathrm{i}}-\boldsymbol{M}^{\mathrm{o}}\right)\right]_{S_{-}^{\mathrm{o}}}^{\text {tan }}=\left[\mathcal{H}\left(\boldsymbol{J}^{\mathrm{o}}, \boldsymbol{M}^{\mathrm{o}}\right)\right]_{S_{+}^{\mathrm{o}}}^{\text {tan }}}
\end{aligned}
$$

where (5) and (6) originate from the definitions of $\boldsymbol{J}^{\mathrm{i}}$ and $\boldsymbol{M}^{\mathrm{i}}$ respectively, and (7) originates from the tangential electric field boundary condition on $S^{g}$, and (8) and (9) originate from the travelling-wave condition on $S^{0}$ (where the travelling-wave condition on output port can be viewed as a counterpart of the famous Sommerfeld's radiation condition at infinity, and a detailed discussion for it can be found in App. A).

Applying the method of moments (MoM) to (5)-(9), the integral equations are discretized into the corresponding matrix equations. The matrix equations imply the linear transformation from independent current $\mathrm{J}^{\mathrm{i}} / \mathrm{M}^{\mathrm{i}}$ into dependent currents $\left(\mathrm{J}^{\mathrm{g}}, \mathrm{J}^{\mathrm{o}}, \mathrm{M}^{\mathrm{i}}, \mathrm{M}^{\mathrm{o}}\right) /\left(\mathrm{J}^{\mathrm{i}}, \mathrm{J}^{\mathrm{g}}, \mathrm{J}^{\mathrm{o}}, \mathrm{M}^{\mathrm{o}}\right)$, and then the following

$$
\left[\begin{array}{l}
\mathrm{J}^{\mathrm{i}} \\
\mathrm{J}^{\mathrm{g}} \\
\mathrm{J}^{\mathrm{o}} \\
\mathrm{M}^{\mathrm{i}} \\
\mathrm{M}^{\mathrm{o}}
\end{array}\right]=\mathrm{T}_{\text {DoJ }} \cdot \mathrm{J}^{\mathrm{i}}=\mathrm{T}_{\text {DoM }} \cdot \mathrm{M}^{\mathrm{i}}
$$

from independent current $\mathrm{J}^{\mathrm{i}} / \mathrm{M}^{\mathrm{i}}$ into all involved currents $\left(\mathrm{J}^{\mathrm{i}}, \mathrm{J}^{\mathrm{g}}, \mathrm{J}^{\mathrm{o}}, \mathrm{M}^{\mathrm{i}}, \mathrm{M}^{\mathrm{o}}\right)$.

Substituting transformation (10) into (4), the following IPO

$$
\begin{aligned}
& P^{\text {in }}=\left(\mathrm{C}^{\mathrm{i}}\right)^{\dagger} \cdot \mathrm{P}^{\mathrm{in}} \cdot \mathrm{C}^{\mathrm{i}}
\end{aligned}
$$

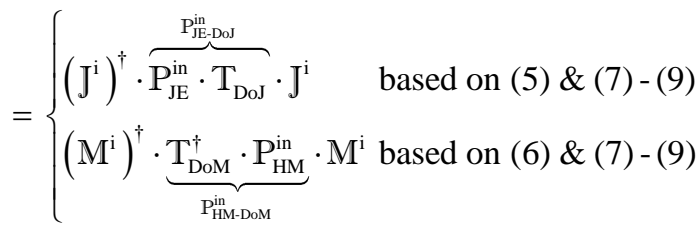

with only independent $\mathrm{C}^{\mathrm{i}}$ (either $\mathrm{J}^{\mathrm{i}}$ or $\mathrm{M}^{\mathrm{i}}$ ) is obtained.

\section{Energy-Decoupled Modes and Their Modal Quantities}

By solving the following modal decoupling equation

$$
\mathrm{P}_{-}^{\text {in }} \cdot \mathrm{C}_{m}^{\mathrm{i}}=\theta_{m} \mathrm{P}_{+}^{\text {in }} \cdot \mathrm{C}_{m}^{\mathrm{i}}
$$

(where $\mathrm{P}_{+}^{\text {in }}=\left[\mathrm{P}^{\text {in }}+\left(\mathrm{P}^{\text {in }}\right)^{\dagger}\right] / 2$ and $\mathrm{P}_{-}^{\text {in }}=\left[\mathrm{P}^{\text {in }}-\left(\mathrm{P}^{\text {in }}\right)^{\dagger}\right] / 2 j$ ) the DMs satisfying the following power-decoupling relation

$$
(1 / 2) \iint_{S^{i}}\left(\boldsymbol{E}_{m} \times \boldsymbol{H}_{n}^{\dagger}\right) \cdot d \boldsymbol{S}=\left(1+j \theta_{m}\right) \delta_{m n}
$$

(which implies time-average power-decoupling relation $\left.(1 / T) \int_{0}^{T}\left[\iint_{S^{\mathrm{i}}}\left(\boldsymbol{\mathcal { E }}_{m} \times \mathcal{H}_{n}\right) \cdot d \boldsymbol{S}\right] d t=\delta_{m n}\right)$ can be obtained.

Following the convention of [54], the modal input impedance and admittance of the $m$-th DM are defined as follows:

$$
\begin{aligned}
& Z_{m}^{\text {in }}=\frac{(1 / 2) \iint_{S^{i}}\left(\boldsymbol{E}_{m} \times \boldsymbol{H}_{m}^{\dagger}\right) \cdot d \boldsymbol{S}}{(1 / 2)\left\langle\boldsymbol{J}_{m}, \boldsymbol{J}_{m}\right\rangle_{S^{\mathrm{i}}}}=\overbrace{\operatorname{Re}\left\{Z_{m}^{\text {in }}\right\}}^{R_{R_{m}^{\text {in }}}^{\text {in }}}+j \overbrace{\operatorname{Im}\left\{Z_{m}^{\text {in }}\right\}}^{X_{m}^{\text {in }}} \\
& Y_{m}^{\text {in }}=\frac{(1 / 2) \iint_{S^{\mathrm{i}}}\left(\boldsymbol{E}_{m} \times \boldsymbol{H}_{m}^{\dagger}\right) \cdot d \boldsymbol{S}}{(1 / 2)\left\langle\boldsymbol{M}_{m}, \boldsymbol{M}_{m}\right\rangle_{S^{\mathrm{i}}}}=\underbrace{\operatorname{Re}\left\{Y_{m}^{\text {in }}\right\}}_{G_{m}^{\text {in }}}+j \underbrace{\operatorname{Im}\left\{Y_{m}^{\text {in }}\right\}}_{B_{m}^{\text {in }}}
\end{aligned}
$$

where $R_{m}^{\text {in }}, X_{m}^{\text {in }}, G_{m}^{\text {in }}$, and $B_{m}^{\text {in }}$ are the corresponding modal input resistance, reactance, conductance, and susceptance.

Evidently, both $R_{m}^{\text {in }}$ and $G_{m}^{\text {in }}$ are the real functions about working frequency $f$, so both the function curves of $R_{m}^{\text {in }}(f)$ and $G_{m}^{\text {in }}(f)$ can be easily obtained. If the following two conditions

C1. frequencies $\left\{f_{m}^{1}, f_{m}^{2}, \cdots, f_{m}^{\xi}, \cdots\right\}$ are all the local maximum points of the $m$-th modal $R_{m}^{\text {in }}(f)$ or $G_{m}^{\text {in }}(f)$ curve and

C2. frequencies $\left\{f_{m}^{1}, f_{m}^{2}, \cdots, f_{m}^{\xi}, \cdots\right\}$ satisfy monotonic relation $f_{m}^{1}<f_{m}^{2}<\cdots<f_{m}^{\xi}<\cdots$

are satisfied simultaneously, then the working frequency $f_{m}^{\xi}$ and cut-off frequency $f_{m}^{c}$ of the $m$-th DM satisfy following

$$
\left(2 \pi f_{m}^{c}\right)^{2} \mu \varepsilon+\left(\frac{2 \pi}{2 L / \xi}\right)^{2}=\left(2 \pi f_{m}^{\xi}\right)^{2} \mu \varepsilon
$$

and then the cut-off frequency $f_{m}^{c}$ can be calculated as the following explicit expression

$$
f_{m}^{\mathrm{c}}=\sqrt{\left(f_{m}^{\xi}\right)^{2}-\left(\frac{\xi}{2 L \sqrt{\mu \varepsilon}}\right)^{2}}
$$

Among all the $\left\{f_{m}^{c}\right\}$, the smallest one corresponds to the dominant mode of the waveguide.

To verify the validities of the theory and formulations established above, the DMs of rectangular (Sec. II-D), circular (Sec. II-E), and coaxial (Sec. II-F) metallic waveguides are constructed by employing the PTT-DMT, and the obtained results are compared with the classical analytical results.

D. Numerical Verification I - Rectangular Metallic Waveguide

In this subsection, the PTT-DMT-based modal analysis for a rectangular metallic waveguide, which is with cross section $20 \mathrm{~mm} \times 10 \mathrm{~mm}$ and infinite longitudinal length and whose tube is with material parameters $\varepsilon_{\mathrm{r}}=2$ and $\mu_{\mathrm{r}}=1$, is done. The PTT-DMT-based calculation doesn't cover whole waveguide, but only a section with longitudinal length $L=100 \mathrm{~mm}$, and the longitudinal infinity feature of the waveguide is modeled by the travelling-wave condition on output port. 


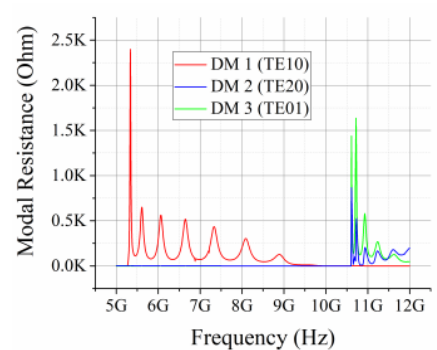

(a)

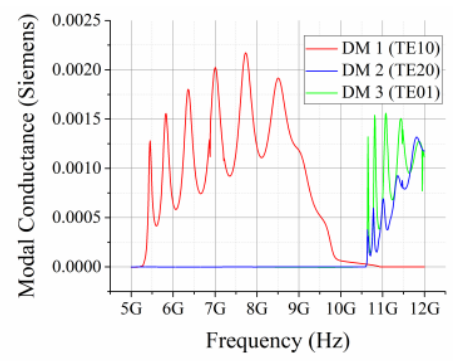

(b)
Fig. 3. Modal input (a) resistance and (b) conductance curves of the first several JE-DoJ-based lower-order DMs of the rectangular metallic waveguide.

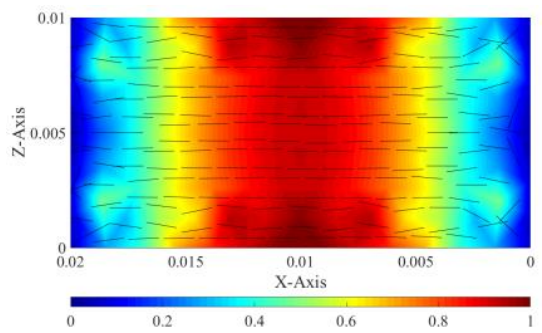

Fig. 4. Modal port magnetic current of the DM 1 working at the first resonance frequency $5.45 \mathrm{GHz}$.

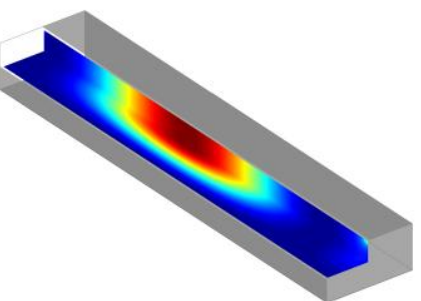

(a)

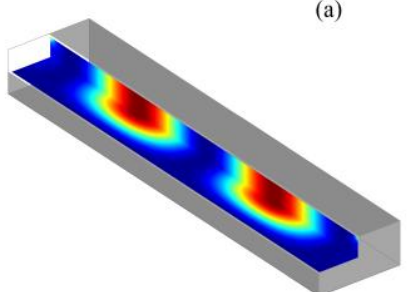

(c)

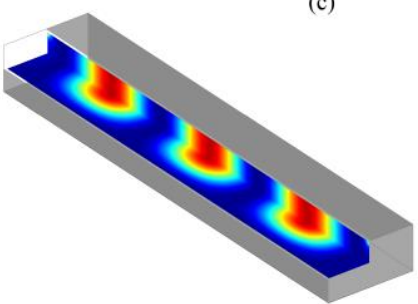

(e)

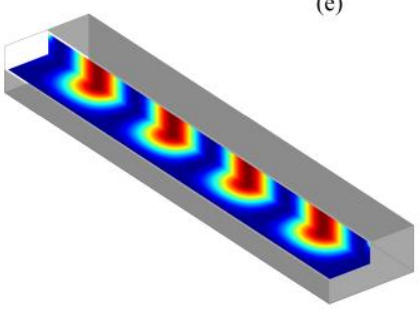

(g)

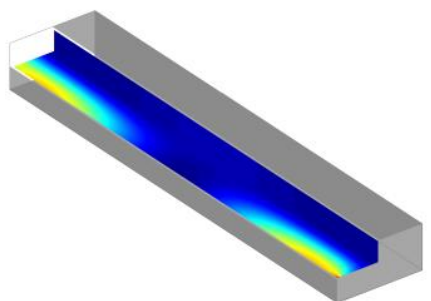

(b)

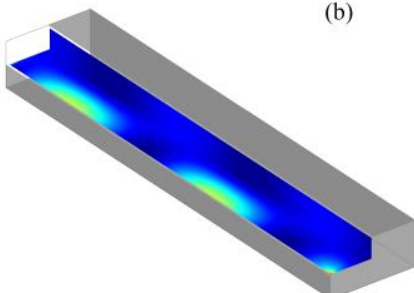

(d)

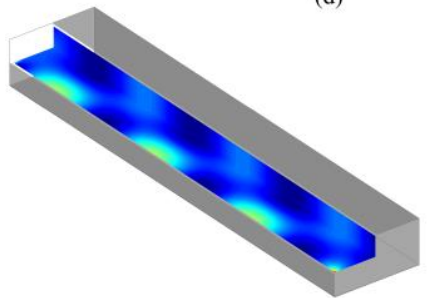

(f)

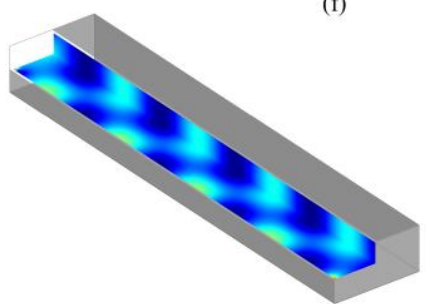

(h)
Fig. 5. (a) Electric energy of the DM 1 at $5.45 \mathrm{GHz}$, (b) magnetic energy of the DM 1 at $5.45 \mathrm{GHz}$, (c) electric energy of the DM 1 at $5.83 \mathrm{GHz}$, (d) magnetic energy of the DM 1 at $5.83 \mathrm{GHz}$, (e) electric energy of the DM 1 at $6.36 \mathrm{GHz}$, (f) magnetic energy of the DM 1 at $6.36 \mathrm{GHz}$, (g) electric energy of the DM 1 at $7.01 \mathrm{GHz}$, and (h) magnetic energy of the DM 1 at $7.01 \mathrm{GHz}$.
The modal resistance and conductance curves of the first several JE-DoJ-based lower-order DMs are shown in Fig. 3, where the JE-DoJ-based DMs are calculated from orthogonalizing the IPO $\mathrm{P}_{\mathrm{JE}-\mathrm{D} \text { of }}^{\mathrm{in}}$ in (11). For the DM 1, its cut-off frequency is calculated as $5.35 \mathrm{GHz}$ by substituting the first resonance frequency $5.45 \mathrm{GHz}$ (corresponding to $\xi=1$ ) into (17), and its modal magnetic current on input port and modal energies corresponding to resonance frequencies $5.45 \mathrm{GHz}, 5.83$ $\mathrm{GHz}, 6.36 \mathrm{GHz}$ and $7.01 \mathrm{GHz}$ are shown in Fig. 4 and Fig. 5. Evidently, the cut-off frequency, current and energy distributions are identical to the ones of the classical TE10 eigen-mode.

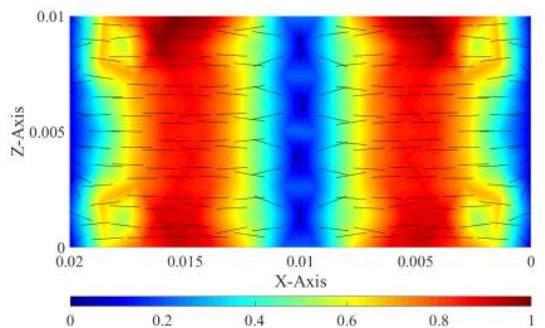

Fig. 6. Modal port magnetic current of the DM 2 working at the first resonance frequency $10.65 \mathrm{GHz}$.

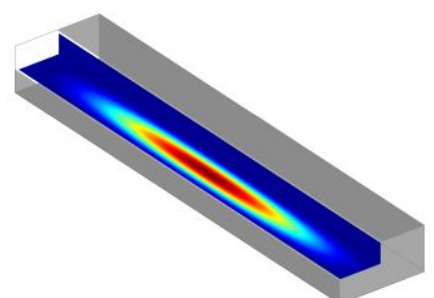

(a)

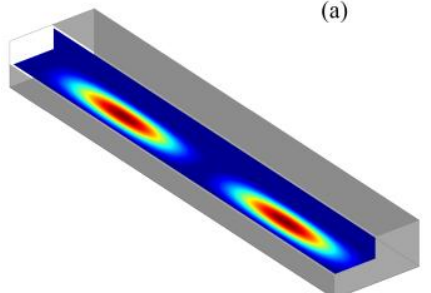

(c)

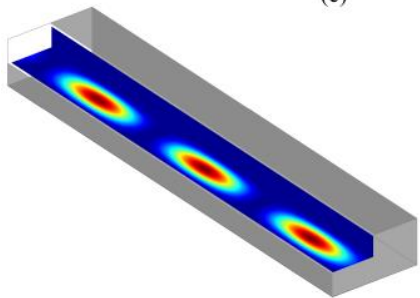

(e)

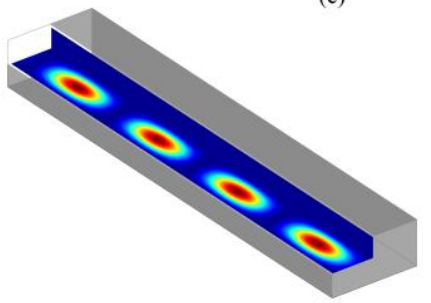

(g)

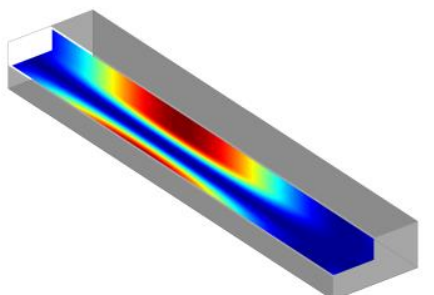

(b)

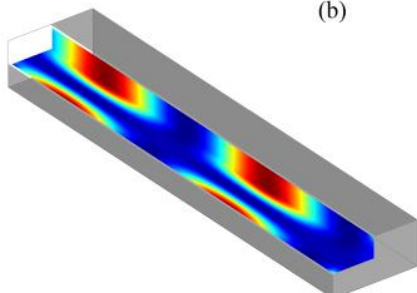

(d)

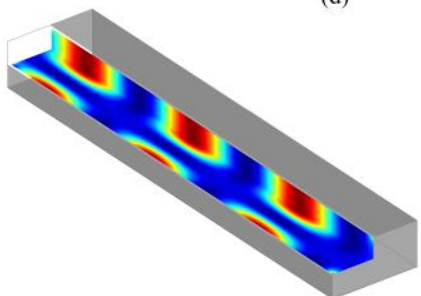

(f)

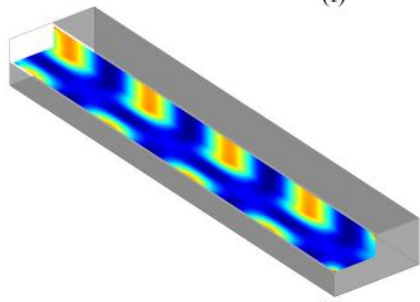

(h)
Fig. 7. (a) Electric energy of the DM 2 at $10.65 \mathrm{GHz}$, (b) magnetic energy of the DM 2 at $10.65 \mathrm{GHz}$, (c) electric energy of the DM 2 at $10.81 \mathrm{GHz}$, (d) magnetic energy of the DM 2 at $10.81 \mathrm{GHz}$, (e) electric energy of the DM 2 at $11.08 \mathrm{GHz}$, (f) magnetic energy of the DM 2 at $11.08 \mathrm{GHz},(\mathrm{g})$ electric energy of the DM 2 at $11.44 \mathrm{GHz}$, and (h) magnetic energy of the DM 2 at $11.44 \mathrm{GHz}$. 
TABLE I CUT-OFF FREQUENCIES (GHZ) AND DEGENERATE DEGREES OF THE FIRST SEVERAL LOWER-ORDER FUNDAMENTAL MODES OF RECTANGULAR WAVEGUIDE DERIVED FROM NOVEL PTT-DMT AND ClASSICAL EMT

\begin{tabular}{|c|c|c|c|}
\hline & \multicolumn{2}{|c|}{ Novel PTT-DMT Established in This Paper } & \multirow[b]{2}{*}{$\begin{array}{c}\text { Classical } \\
\text { EMT [2]-[5] }\end{array}$} \\
\hline & $\begin{array}{l}\text { Recognized from the } \\
\text { Local Maximum of } \\
\text { Resistance Curve }\end{array}$ & $\begin{array}{l}\text { Recognized from the } \\
\text { Local Maximum of } \\
\text { Conductance Curve }\end{array}$ & \\
\hline TE10 & $05.23(1)$ & $05.35(1)$ & $05.30(1)$ \\
\hline TE20 & $10.56(1)$ & $10.60(1)$ & $10.60(1)$ \\
\hline TE01 & $10.56(1)$ & $10.61(1)$ & $10.60(1)$ \\
\hline TE11 & $11.81(1)$ & $11.84(1)$ & $11.85(1)$ \\
\hline TM11 & $11.85(1)$ & $11.84(1)$ & $11.85(1)$ \\
\hline TE21 & $14.96(1)$ & $14.98(1)$ & $14.99(1)$ \\
\hline TM21 & $14.98(1)$ & $14.96(1)$ & $14.99(1)$ \\
\hline
\end{tabular}

For the DM 2 in Fig. 3(b), its cut-off frequency is calculated as $10.60 \mathrm{GHz}$ by substituting the first resonance frequency $10.65 \mathrm{GHz}$ (corresponding to $\xi=1$ ) into (17), and its modal magnetic current on input port and modal energies corresponding to resonance frequencies $10.65 \mathrm{GHz}, 10.81 \mathrm{GHz}$, $11.08 \mathrm{GHz}$ and $11.44 \mathrm{GHz}$ are shown in Fig. 7 and Fig. 8. Evidently, the cut-off frequency, current and energies are identical to the ones of the classical TE20 eigen-mode.

Similarly, it can be found out that the DM 3 shown in Fig. 3 (b) is identical to the classical TE01 eigen-mode. The cut-off frequencies of the first several lower-order DMs (derived from PTT-DMT) and eigen-modes (derived from classical EMT) are listed in Tab. I. Evidently, the results derived from the different theories are consistent with each other except some small numerical errors. In addition, it is easy to observe that: for the TE modes, the cut-off frequencies calculated from conductance curves are more precise; for the TM modes, the cut-off frequencies calculated from resistance curves are more precise.

\section{E. Numerical Verification II - Circular Metallic Waveguide}

In this subsection, the PTT-DMT-based modal analysis for a circular metallic waveguide, which is with cross-section radius $15 \mathrm{~mm}$ and infinite longitudinal length and whose tube is with $\varepsilon_{\mathrm{r}}=2$ and $\mu_{\mathrm{r}}=1$, is done, and the PTT-DMT-based calculation only covers a section with longitudinal length $100 \mathrm{~mm}$ (the longitudinal infinity feature of the waveguide is modeled by the travelling-wave condition on output port).

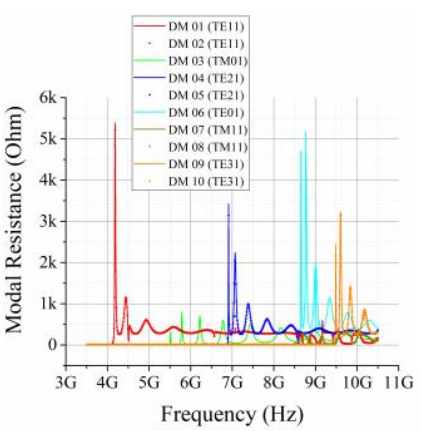

(a) (b)

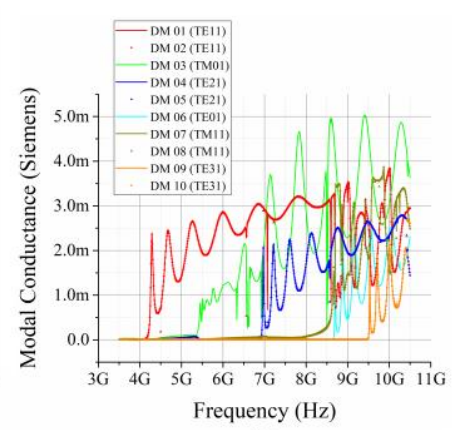

Fig. 8. Modal input (a) resistance and (b) conductance curves of the first several JE-DoJ-based lower-order DMs of the circular metallic waveguide.
TABLE II CUT-OFF FREQUENCIES (GHZ) AND DEGENERATE DEGREES OF THE FIRST SEVERAL LOWER-ORDER FUNDAMENTAL MODES OF CIRCULAR WAVEGUIDE DERIVED FROM NOVEL PTT-DMT AND CLASSICAL EMT

\begin{tabular}{|c|c|c|c|}
\hline & \multicolumn{2}{|c|}{ Novel PTT-DMT Established in This Paper } & \multirow[b]{2}{*}{$\begin{array}{c}\text { Classical } \\
\text { EMT [2]-[5] }\end{array}$} \\
\hline & $\begin{array}{l}\text { Recognized from the } \\
\text { Local Maximum of } \\
\text { Resistance Curve }\end{array}$ & $\begin{array}{l}\text { Recognized from the } \\
\text { Local Maximum of } \\
\text { Conductance Curve }\end{array}$ & \\
\hline TE11 & $04.05(2)$ & $04.16(2)$ & $04.14(2)$ \\
\hline TM01 & $05.41(1)$ & $05.41(1)$ & $05.41(1)$ \\
\hline TE21 & $06.83(2)$ & $06.89(2)$ & $06.87(2)$ \\
\hline TE01 & $08.59(1)$ & $08.64(1)$ & $08.62(1)$ \\
\hline TM11 & $08.64(2)$ & $08.59(2)$ & $08.62(2)$ \\
\hline TE31 & $09.43(2)$ & $09.47(2)$ & $09.45(2)$ \\
\hline TM21 & $11.57(2)$ & $11.55(2)$ & $11.55(2)$ \\
\hline
\end{tabular}

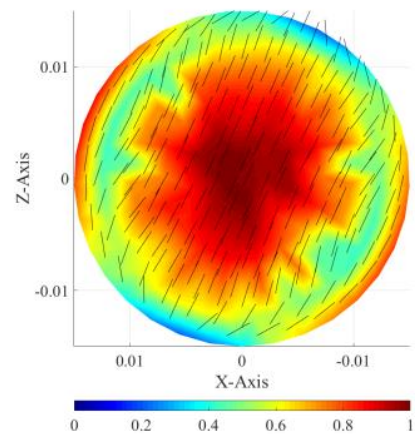

(a)

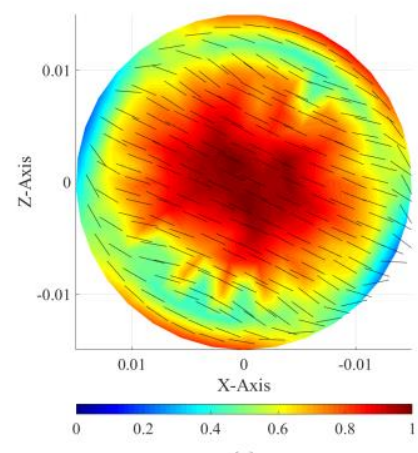

(c)

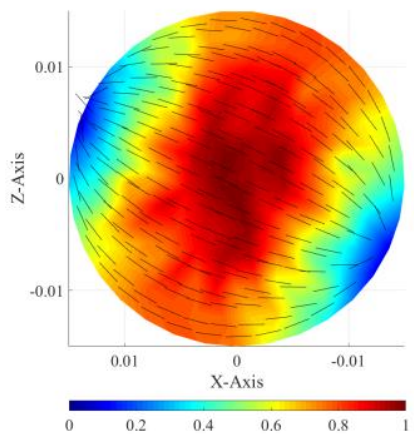

(b)

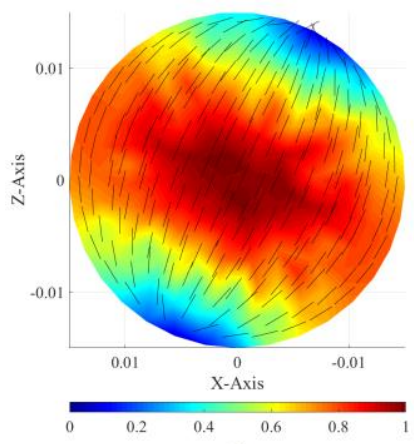

(d)
Fig. 9. Modal port (a) electric current of the DM 1 at $4.29 \mathrm{GHz}$, (b) magnetic current of the DM 1 at $4.29 \mathrm{GHz}$, (c) electric current of the DM 2 at $4.29 \mathrm{GHz}$, and (d) magnetic current of the DM 2 at $4.29 \mathrm{GHz}$.

The modal input resistance and conductance curves associated to the first several JE-DoJ-based lower-order DMs are shown in Fig. 8. The cut-off frequencies of the first several lower-order DMs (derived from novel PTT-DMT) and eigen-modes (derived from classical EMT) are listed in Tab. II.

For the DM 1 and DM 2 in Fig. 8(b), their cut-off frequencies are calculated as $4.16 \mathrm{GHz}$ by substituting the first resonance frequency $4.29 \mathrm{GHz}$ into (17), and their modal currents on input port are shown in Fig. 9. Evidently, the cut-off frequency and currents are identical to the two degenerate states of the classical TE11 eigen-mode. In addition, the modal energies corresponding to the resonance frequencies $4.29 \mathrm{GHz}, 4.68 \mathrm{GHz}$, $5.26 \mathrm{GHz}$ and $6.00 \mathrm{GHz}$ of the first degenerate state are shown in Fig. 10. 


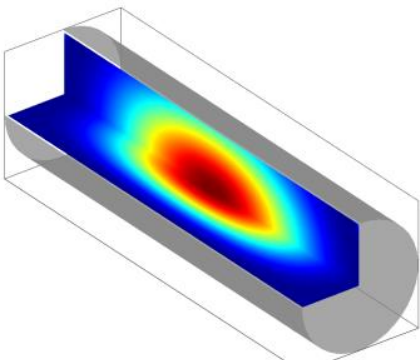

(a)

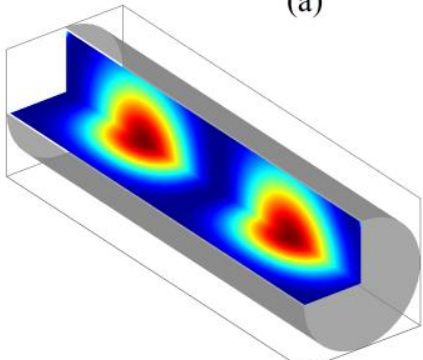

(c)

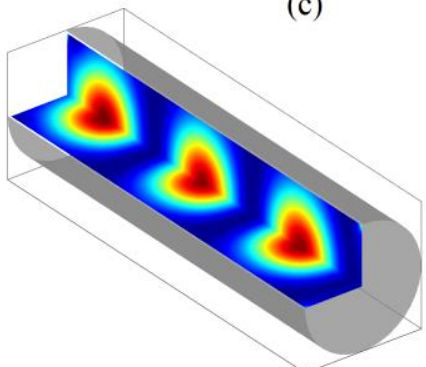

(e)

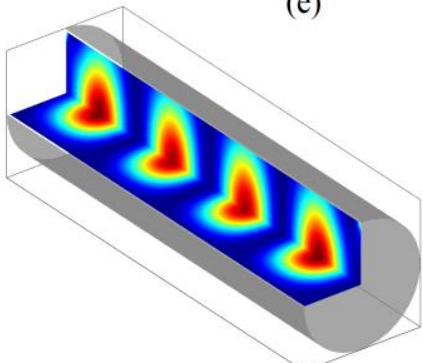

(g)

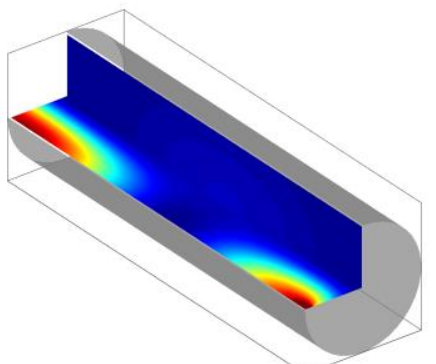

(b)

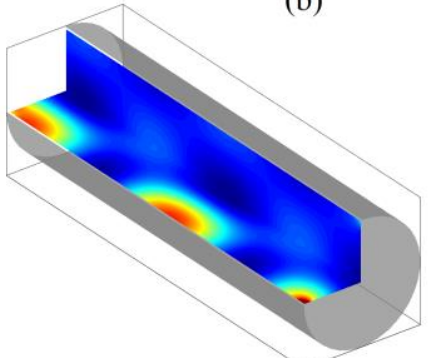

(d)

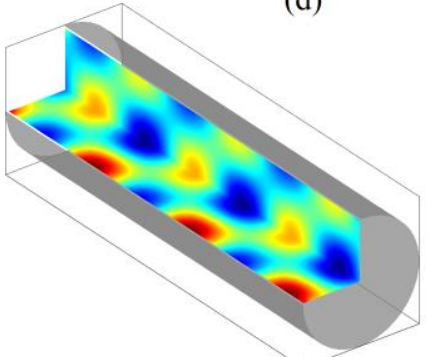

(f)

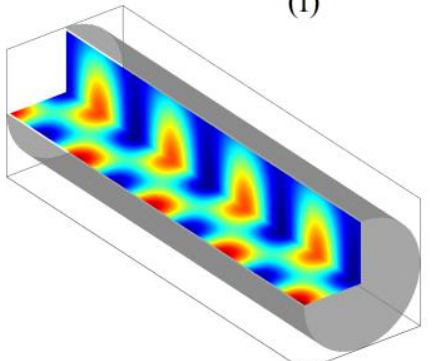

(h)

Fig. 10. (a) Electric energy of the DM 1 at $4.29 \mathrm{GHz}$, (b) magnetic energy of the DM 1 at $4.29 \mathrm{GHz}$, (c) electric energy of the DM 1 at $4.68 \mathrm{GHz}$, (d) magnetic energy of the DM 1 at $4.68 \mathrm{GHz}$, (e) electric energy of the DM 1 at $5.26 \mathrm{GHz}$, (f) magnetic energy of the DM 1 at $5.26 \mathrm{GHz}$, (g) electric energy of the DM 1 at $6.00 \mathrm{GHz}$, and (h) magnetic energy of the DM 1 at $6.00 \mathrm{GHz}$.

For the DM 3, its modal electric and magnetic currents distributing on input port are shown in Fig. 11.

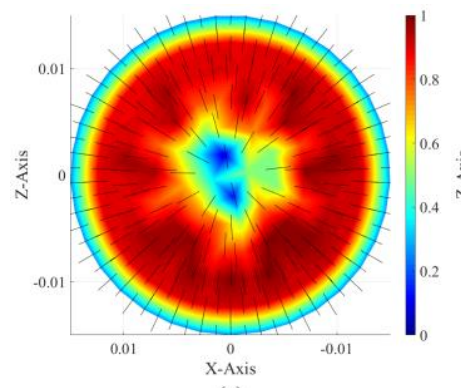

(a)

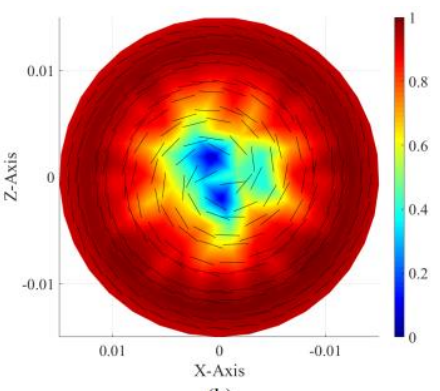

(b)

Fig. 11. Modal port (a) electric and (b) magnetic currents of the DM 3 working at $5.52 \mathrm{GHz}$.

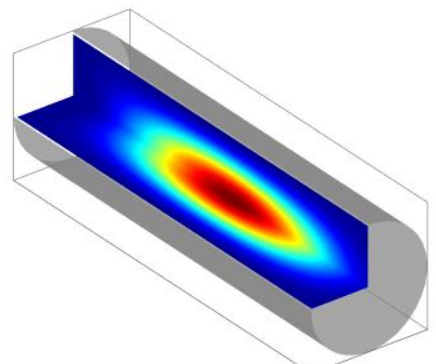

(a)

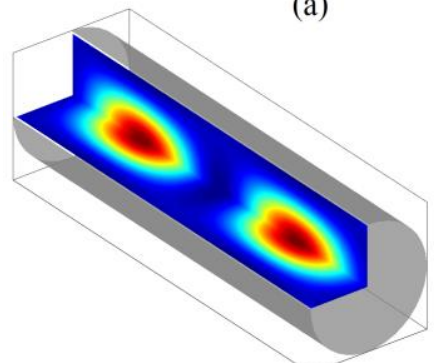

(c)

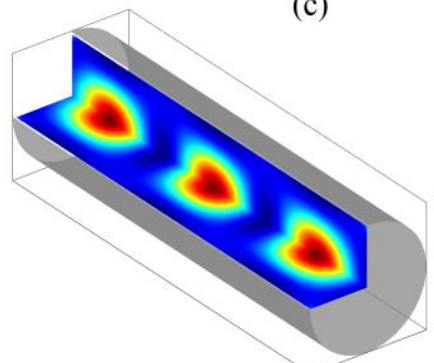

(e)

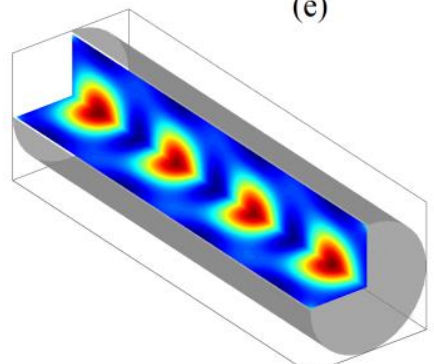

(g)

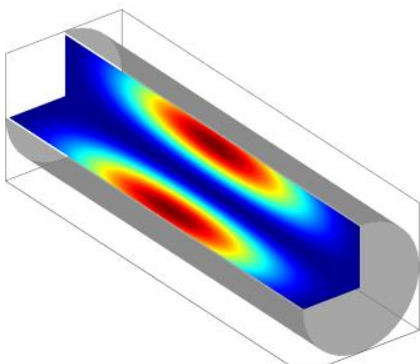

(b)

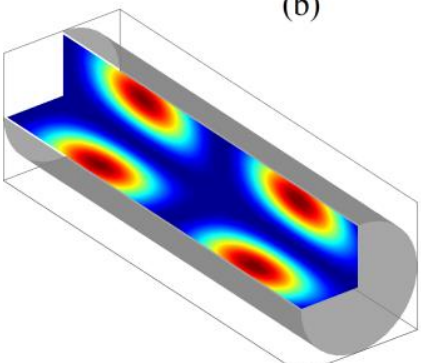

(d)

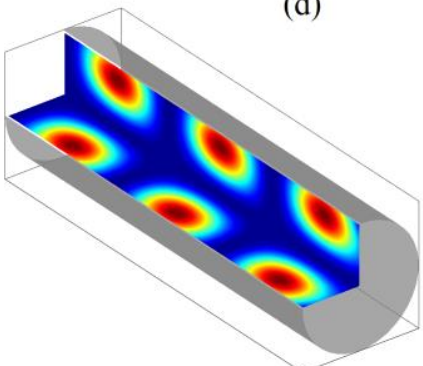

(f)

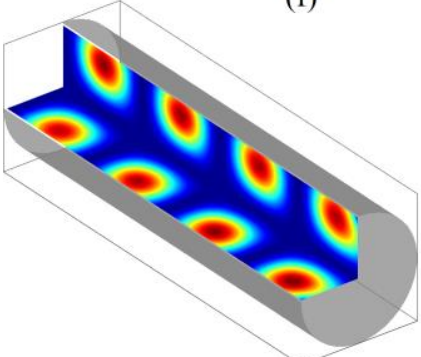

(h)

Fig. 12. (a) Electric energy of the DM 3 at $5.52 \mathrm{GHz}$, (b) magnetic energy of the DM 3 at $5.52 \mathrm{GHz}$, (c) electric energy of the DM 3 at $5.79 \mathrm{GHz}$, (d) magnetic energy of the DM 3 at $5.79 \mathrm{GHz}$, (e) electric energy of the DM 3 at $6.22 \mathrm{GHz}$, (f) magnetic energy of the DM 3 at $6.22 \mathrm{GHz}$, (g) electric energy of the DM 3 at $6.78 \mathrm{GHz}$, and (h) magnetic energy of the DM 3 at $6.78 \mathrm{GHz}$.

Evidently, the mode is rotationally symmetrical about Y-axis, so it has only one degenerate state. The cut-off frequency and current distributions are identical to the ones of classical TM01 eigen-mode. In addition, the modal electric and magnetic energy distributions corresponding to the resonance frequencies $5.52 \mathrm{GHz}, 5.79 \mathrm{GHz}, 6.22 \mathrm{GHz}$ and $6.78 \mathrm{GHz}$ of the only degenerate state are shown in Fig. 12.

Similarly, it can be found out that the DM 4 DM 10 are just the classical TE21 eigen-mode (the first degenerate state), TE21 eigen-mode (the second degenerate state), TE01 eigen-mode, TM11 eigen-mode (the first degenerate state), TM11 eigen-mode (the second degenerate state), TE31 eigen-mode (the first degenerate state) and TE31 eigen-mode (the second degenerate state) respectively. 
For facilitating readers' comparisons and references, the cut-off frequencies of the above-mentioned modes are listed in Tab. II, and the port currents of DMs 1 10 are shown in Fig. 13, which are consistent with the ones shown in [4, Fig. 9-2].
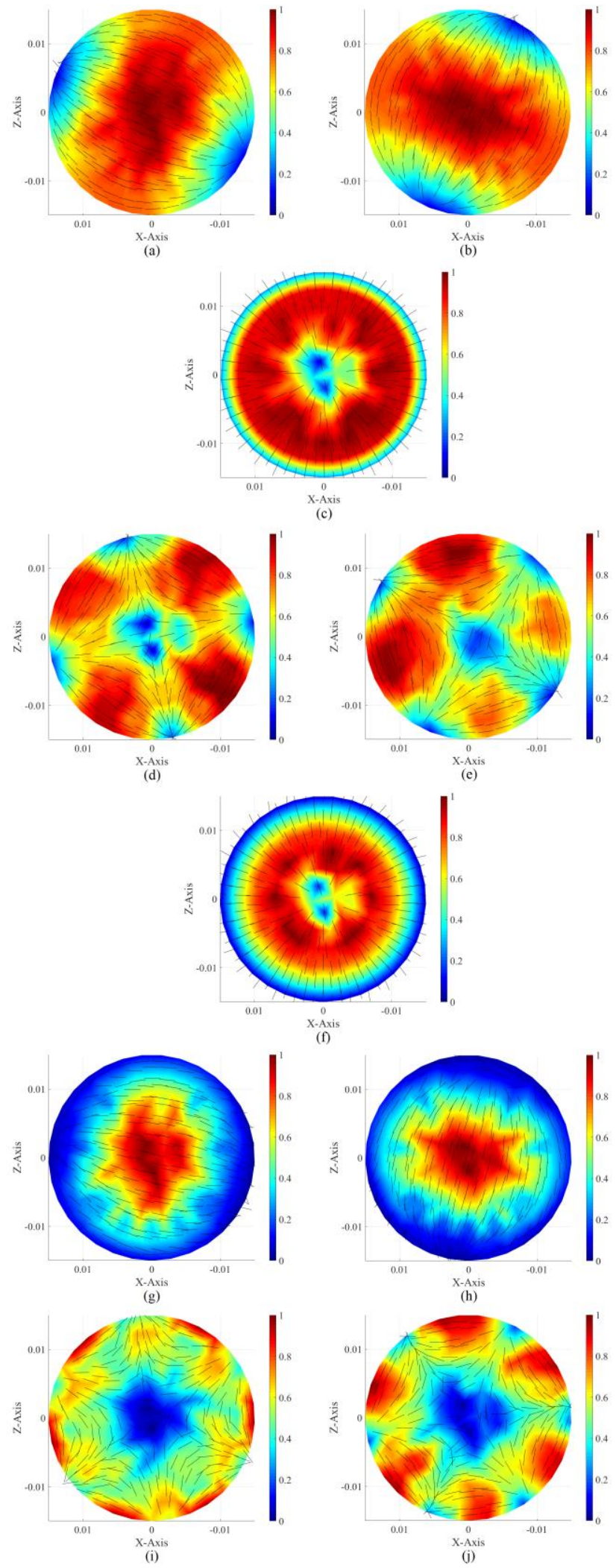

Fig. 13. Modal port (a) $\boldsymbol{M}^{\mathrm{i}}$ of DM 1, (b) $\boldsymbol{M}^{\mathrm{i}}$ of DM 2, (c) $\boldsymbol{J}^{\mathrm{i}}$ of DM 3, (d) $\boldsymbol{M}^{\mathrm{i}}$ of DM 4, (e) $\boldsymbol{M}^{\mathrm{i}}$ of DM 5, (f) $\boldsymbol{M}^{\mathrm{i}}$ of DM 6, (g) $\boldsymbol{J}^{\mathrm{i}}$ of DM 7, (h) $\boldsymbol{J}^{\mathrm{i}}$ of DM 8, (i) $\boldsymbol{M}^{\text {i }}$ of DM 9, and (j) $\boldsymbol{M}^{\text {i }}$ of DM 10.

\section{F. Numerical Verification III - Coaxial Metallic Waveguide}

In this subsection, the PTT-DMT-based modal analysis for a coaxial metallic waveguide, which is with inner radius $2 \mathrm{~mm}$, outer radius $7 \mathrm{~mm}$ and infinite longitudinal length and whose tube is filled with $\varepsilon_{\mathrm{r}}=2$ and $\mu_{\mathrm{r}}=1$, is done, and the PTT-DMT-based calculation only covers a section with longitudinal length $50 \mathrm{~mm}$ (the longitudinal infinity feature of the waveguide is modeled by the travelling-wave condition on output port).

The modal input resistance curve associated to the JE-DoJ-based dominant DM is shown in Fig. 14.

For the DM shown in Fig. 14, its modal electric and magnetic currents distributing on input port are shown in Fig. 15, and they are identical to the ones of the classical TEM eigen-mode. We calculate the TEM working frequencies corresponding to $L=\lambda_{z} / 2, L=2 \lambda_{z} / 2, L=3 \lambda_{z} / 2$, and $L=4 \lambda_{z} / 2$ from the novel PTT-DMT built in this paper and the classical EMT [2]-[5], and list them in Tab. III. In addition, we also show the modal electric and magnetic energy distributions corresponding to $2.06 \mathrm{GHz}, 4.12 \mathrm{GHz}, 6.18 \mathrm{GHz}$, and $8.23 \mathrm{GHz}$ in Fig. 16

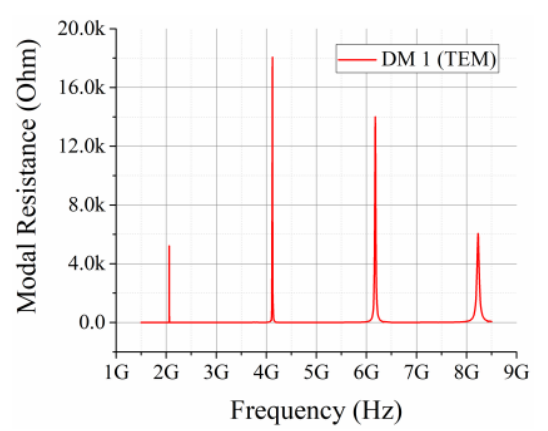

Fig. 14. Modal input resistance curve of the JE-DoJ-based dominant DM.

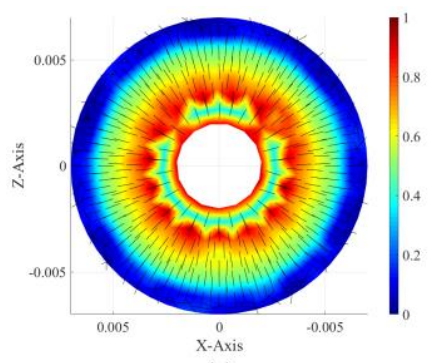

(a)

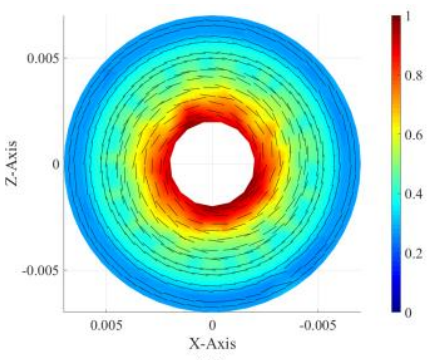

(b)
Fig. 15. Modal port (a) electric and (b) magnetic currents of the dominant DM working at $2.06 \mathrm{GHz}$.

TABLE III WORKING FREQUENCIES (GHz) AND DEGENERATE DEGREES OF THE TEM Mode of CiRCUlaR COAXIAL WAVEGUIDE DERIVED FROM NOVEL PTT-DMT AND CLASSICAL EMT

\begin{tabular}{|c|c|c|}
\hline \hline & $\begin{array}{c}\text { Novel PTT-DMT } \\
\text { Built in This Paper }\end{array}$ & $\begin{array}{c}\text { Classical EMT } \\
\text { [2]-[5] }\end{array}$ \\
\hline $\begin{array}{c}\text { Working Frequency } \\
\text { Corresponding to } \boldsymbol{L}=\mathbf{1} \boldsymbol{\lambda}_{z} / \mathbf{2}\end{array}$ & $2.06(1)$ & $2.12(1)$ \\
\hline $\begin{array}{c}\text { Working Frequency } \\
\text { Corresponding to } \boldsymbol{L}=\mathbf{2} \boldsymbol{\lambda}_{z} / \mathbf{2}\end{array}$ & $4.12(1)$ & $4.24(1)$ \\
\hline $\begin{array}{c}\text { Working Frequency } \\
\text { Corresponding to } \boldsymbol{L}=\mathbf{3} \boldsymbol{\lambda}_{z} / \mathbf{2}\end{array}$ & $6.18(1)$ & $6.36(1)$ \\
\hline $\begin{array}{c}\text { Working Frequency } \\
\text { Corresponding to } \boldsymbol{L}=\mathbf{4} \lambda_{z} / \mathbf{2}\end{array}$ & $8.23(1)$ & $8.48(1)$ \\
\hline \hline
\end{tabular}




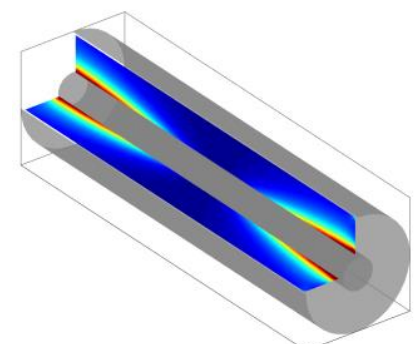

(a)

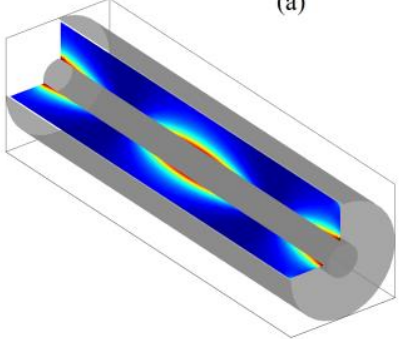

(c)

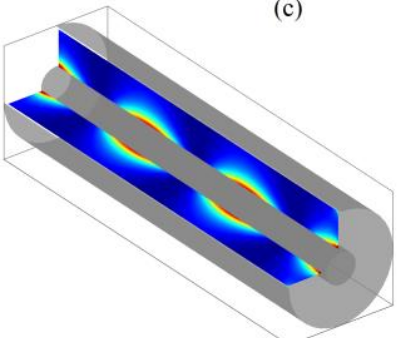

(e)

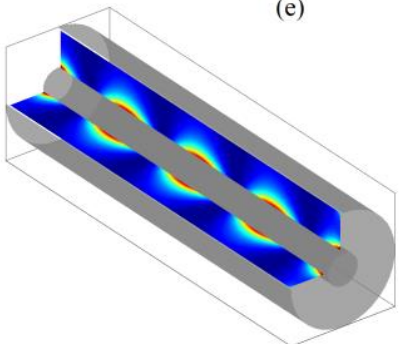

(g)

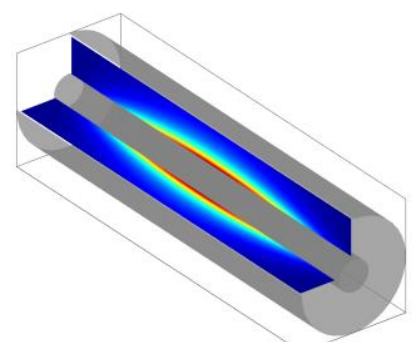

(b)

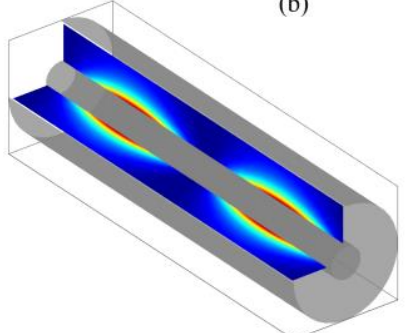

(d)

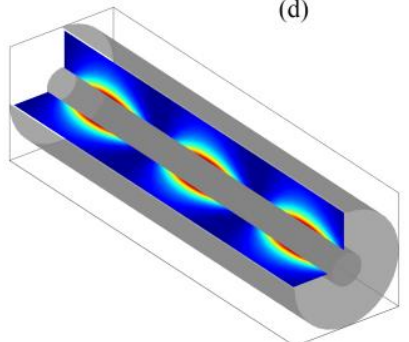

(f)

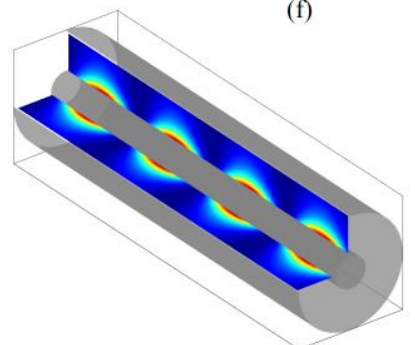

(h)
Fig. 16. (a) Electric energy of the DM 1 at $2.06 \mathrm{GHz}$, (b) magnetic energy of the $\mathrm{DM} 1$ at $2.06 \mathrm{GHz}$, (c) electric energy of the DM 1 at $4.12 \mathrm{GHz}$, (d) magnetic energy of the DM 1 at $4.12 \mathrm{GHz}$, (e) electric energy of the DM 1 at $6.18 \mathrm{GHz}$, (f) magnetic energy of the $\mathrm{DM} 1$ at $6.18 \mathrm{GHz},(\mathrm{g})$ electric energy of the DM 1 at $8.23 \mathrm{GHz}$, and (h) magnetic energy of the DM 1 at $8.23 \mathrm{GHz}$.

\section{PTT-DMT For MATERIAL WAVEGUIDES}

This section is devoted to further generalizing the idea of Sec. II to material waveguides. A section $V$ of material waveguide is shown in Fig. 17 (where the whole waveguide is placed in free-space environment), and its material parameters are $(\mu, \varepsilon)$. The input and output ports of $V$ are denoted as $S^{\mathrm{i}}$ and $S^{\circ}$ respectively, and the interface between $V$ and environment is denoted as $S^{\mathrm{f}}$, as shown in Fig. 17.

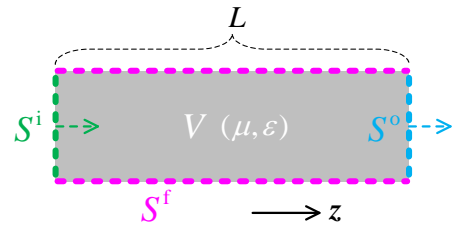

Fig. 17. Topological structure of a material waveguide section with longitudinal length $L$.

\section{A. Core Formulations}

If the equivalent currents on $S^{\mathrm{i}}, S^{\mathrm{f}}$, and $S^{\mathrm{o}}$ are denoted as $\left(\boldsymbol{J}^{\mathrm{i}}, \boldsymbol{M}^{\mathrm{i}}\right),\left(\boldsymbol{J}^{\mathrm{f}}, \boldsymbol{M}^{\mathrm{f}}\right)$, and $\left(\boldsymbol{J}^{\mathrm{o}}, \boldsymbol{M}^{\mathrm{o}}\right)$ respectively, then the electric and magnetic fields distributing on $V$ can be obtained by acting operators $\mathcal{E}$ and $\mathcal{H}$ on the currents respectively, so the IPO of $V$ is as follows:

$$
\begin{aligned}
P^{\text {in }} & =-(1 / 2)\left\langle\boldsymbol{J}^{\mathrm{i}}, \mathcal{E}\left(\boldsymbol{J}^{\mathrm{i}}+\boldsymbol{J}^{\mathrm{f}}-\boldsymbol{J}^{\mathrm{o}}, \boldsymbol{M}^{\mathrm{i}}+\boldsymbol{M}^{\mathrm{f}}-\boldsymbol{M}^{\mathrm{o}}\right)\right\rangle_{S_{+}^{\mathrm{i}}} \\
& =-(1 / 2)\left\langle\boldsymbol{M}^{\mathrm{i}}, \mathcal{H}\left(\boldsymbol{J}^{\mathrm{i}}+\boldsymbol{J}^{\mathrm{f}}-\boldsymbol{J}^{\mathrm{o}}, \boldsymbol{M}^{\mathrm{i}}+\boldsymbol{M}^{\mathrm{f}}-\boldsymbol{M}^{\mathrm{o}}\right)\right\rangle_{S_{+}^{\mathrm{i}}}^{\dagger}
\end{aligned}
$$

where the first and second lines correspond to the JE and HM forms respectively. The basis function expansions for the currents discretize the JE and HM integral forms of IPO into the following matrix forms

$$
P^{\text {in }}=\left(\mathrm{J}^{\mathrm{i}}\right)^{\dagger} \cdot \mathrm{P}_{\mathrm{JE}}^{\mathrm{in}} \cdot\left[\begin{array}{l}
\mathrm{J}^{\mathrm{i}} \\
\mathrm{J}^{\mathrm{f}} \\
\mathrm{J}^{\mathrm{o}} \\
\mathrm{M}^{\mathrm{i}} \\
\mathrm{M}^{\mathrm{f}} \\
\mathrm{M}^{\mathrm{o}}
\end{array}\right]=\left[\begin{array}{l}
\mathrm{J}^{\mathrm{i}} \\
\mathrm{J}^{\mathrm{f}} \\
\mathrm{J}^{\mathrm{o}} \\
\mathrm{M}^{\mathrm{i}} \\
\mathrm{M}^{\mathrm{f}} \\
\mathrm{M}^{\mathrm{o}}
\end{array}\right]^{\dagger} \cdot \mathrm{P}_{\mathrm{HM}}^{\mathrm{in}} \cdot \mathrm{M}^{\mathrm{i}}
$$

with both independent current $\mathrm{J}^{\mathrm{i}} / \mathrm{M}^{\mathrm{i}}$ and dependent currents $\left(J^{\mathrm{f}}, \mathrm{J}^{\mathrm{o}}, \mathrm{M}^{\mathrm{i}}, \mathrm{M}^{\mathrm{f}}, \mathrm{M}^{\mathrm{o}}\right) /\left(\mathrm{J}^{\mathrm{i}}, \mathrm{J}^{\mathrm{f}}, \mathrm{J}^{\mathrm{o}}, \mathrm{M}^{\mathrm{f}}, \mathrm{M}^{\mathrm{o}}\right)$.

In fact, the above-mentioned currents are not independent, and there exists a linear transformation from the independent current $\mathrm{J}^{\mathrm{i}} / \mathrm{M}^{\mathrm{i}}$ into the other dependent currents $\left(\mathrm{J}^{\mathrm{f}}, \mathrm{J}^{\mathrm{o}}, \mathrm{M}^{\mathrm{i}}, \mathrm{M}^{\mathrm{f}}, \mathrm{M}^{\mathrm{o}}\right) /\left(\mathrm{J}^{\mathrm{i}}, \mathrm{J}^{\mathrm{f}}, \mathrm{J}^{\mathrm{o}}, \mathrm{M}^{\mathrm{f}}, \mathrm{M}^{\mathrm{o}}\right)$. The detailed formulations for establishing the transformation are provided in the App. B. Applying the transformation to (19), the IPO $P^{\text {in }}=\left(\mathrm{C}^{\mathrm{i}}\right)^{\dagger} \cdot \mathrm{P}^{\text {in }} \cdot \mathrm{C}^{\mathrm{i}}$ with only independent current $\mathrm{C}^{\mathrm{i}}$ (which is either $\mathrm{J}^{\mathrm{i}}$ or $\mathrm{M}^{\mathrm{i}}$ ) is obtained. By orthogonalizing IPO $\mathrm{P}^{\text {in }}$, the DMs of the material waveguide can be derived from solving modal decoupling equation $\mathrm{P}_{-}^{\text {in }} \cdot \mathrm{C}_{m}^{\mathrm{i}}=\theta_{m} \mathrm{P}_{+}^{\text {in }} \cdot \mathrm{C}_{m}^{\mathrm{i}}$. By employing the method used in Sec. II-C, the travelling-wave DMs can be recognized, and their cut-off frequencies can be calculated.

\section{B. Numerical Verifications}

In this subsection, the PTT-DMT-based modal analysis for a circular dielectric rod waveguide, which is with radius $5 \mathrm{~mm}$, infinite longitudinal length and material parameters $\left(\varepsilon_{\mathrm{r}}=10, \mu_{\mathrm{r}}=1\right)$, is done. The PTT-DMT-based calculation doesn't cover whole waveguide, but only a section with longitudinal length $L=25 \mathrm{~mm}$, and the longitudinal infinity feature of the waveguide is modeled by the travelling-wave condition on output port.

The modal input conductance curves associated to the first several HM-DoM-based lower-order DMs are shown in Fig. 18, where the HM-DoM-based DMs are calculated from orthogonalizing the IPO $\mathrm{P}_{\mathrm{HM}}^{\text {in }}$ given in (19) with the transformation from $\mathrm{M}^{\mathrm{i}}$ into $\left(\mathrm{J}^{\mathrm{i}}, \mathrm{J}^{\mathrm{f}}, \mathrm{J}^{\mathrm{o}}, \mathrm{M}^{\mathrm{f}}, \mathrm{M}^{\mathrm{o}}\right)$. 


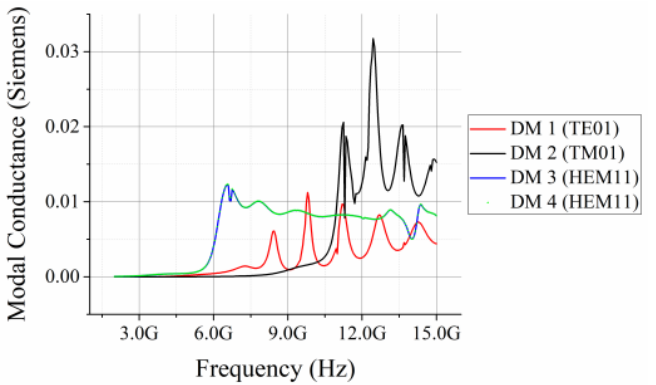

Fig. 18. Modal input conductance curves of the first several HM-DoM-based lower-order DMs of the circular material waveguide.

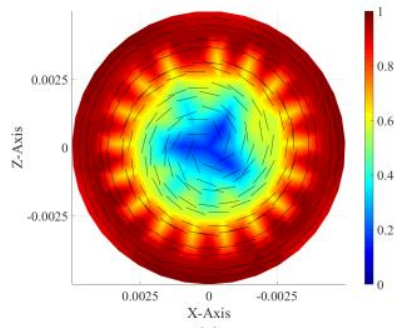

(a)

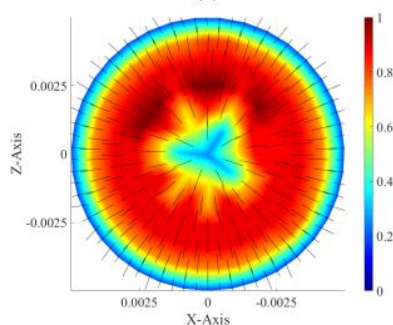

(c)

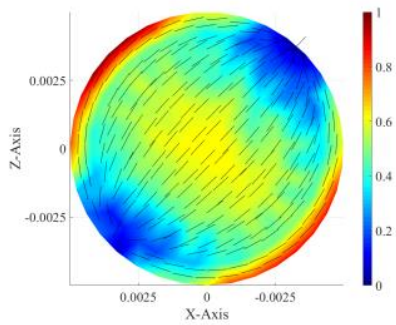

(e)

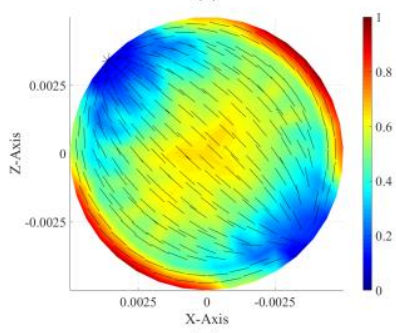

(g)

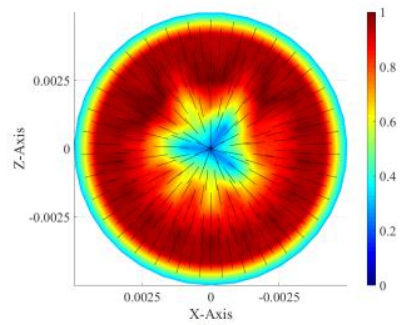

(b)

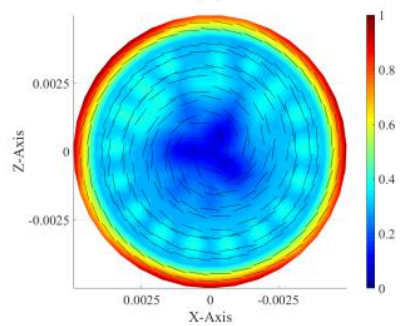

(d)

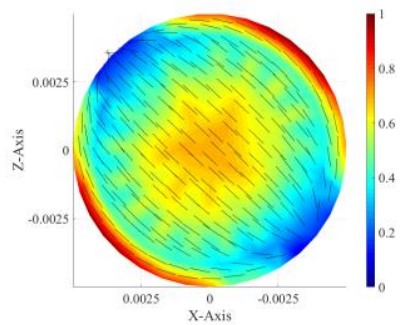

(f)

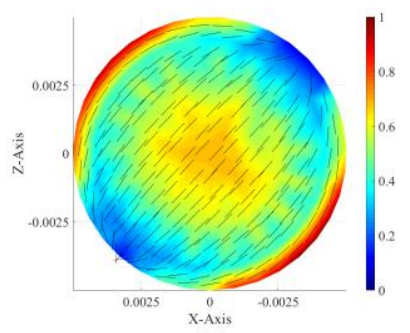

(h)
Fig. 19. Modal port (a) electric current of DM 1, (b) magnetic current of DM 1, (c) electric current of DM 2, (d) magnetic current of DM 2, (e) electric current of DM 3, (f) magnetic current of DM 3, (g) electric current of DM 4, and (h) magnetic current of DM 4.

For the DMs shown in Fig. 18, their modal electric and magnetic currents on input port are shown in Fig. 19. From the currents, it is easy to find out that: the DM 1 is TE01 eigen-mode; the DM 2 is TM01 eigen-mode; the DMs 3 and 4 are the two degenerate states of HEM11 eigen-mode. For the DM 1 (TE01 eigen-mode), its modal electric and magnetic energy distributions corresponding to working frequencies $7.25 \mathrm{GHz}$, 8.45 GHz, $9.80 \mathrm{GHz}$, and $11.20 \mathrm{GHz}$ are shown in Fig. 20.

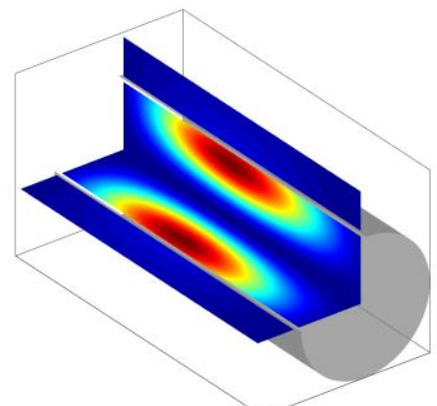

(a)

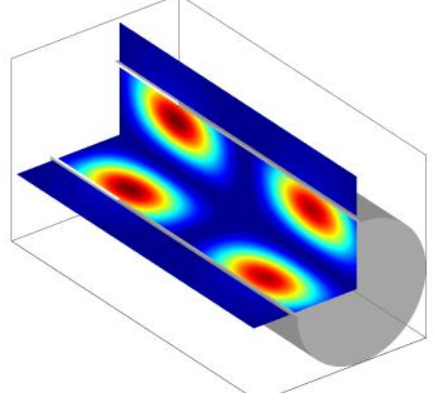

(c)

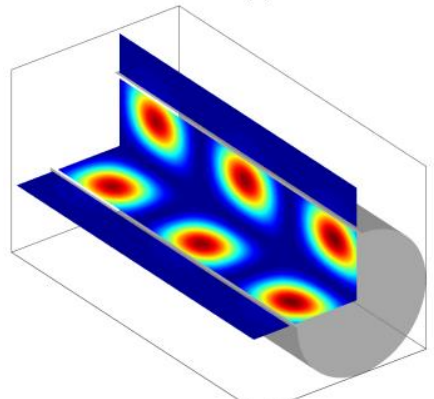

(e)

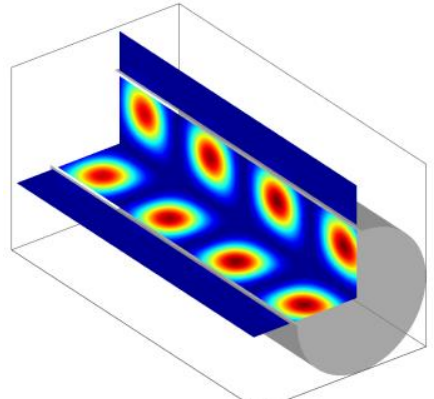

(g)

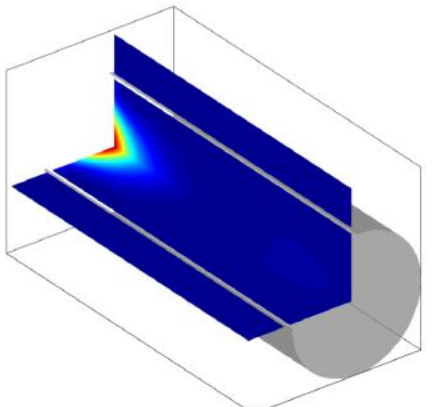

(b)

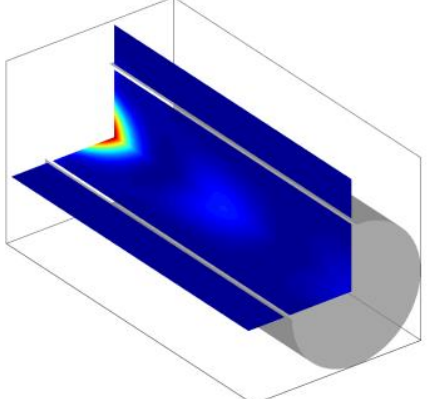

(d)

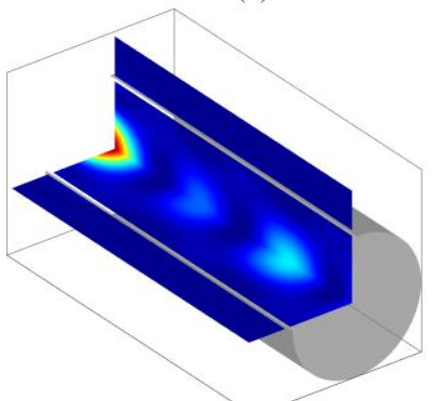

(f)

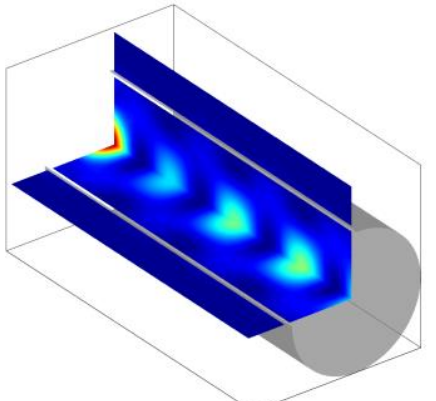

(h)
Fig. 20. (a) Electric energy of the DM 1 at $7.25 \mathrm{GHz}$, (b) magnetic energy of the DM 1 at $7.25 \mathrm{GHz}$, (c) electric energy of the DM 1 at $8.45 \mathrm{GHz}$, (d) magnetic energy of the DM 1 at $8.45 \mathrm{GHz}$, (e) electric energy of the DM 1 at $9.80 \mathrm{GHz}$, (f) magnetic energy of the DM 1 at $9.80 \mathrm{GHz}$, (g) electric energy of the DM 1 at $11.20 \mathrm{GHz}$, and (h) magnetic energy of the DM 1 at $11.20 \mathrm{GHz}$.

\section{CONCLUDING REMARKS}

Just like the wave-port-fed transmitting antenna discussed by us previously, a section of any wave-guiding structure (either metallic or material waveguide) also contains an input port as the entrance for EM energy, an output port as the exit for EM energy, and a waveguide wall for guiding EM energy from the input port to the output port. Besides governing the transport 
process of the power-flow passing through an antenna, PTT also governs the transport process of the power-flow passing through the waveguide section. Besides sustaining a stationary power transport related to antenna, the IPO, which is the excitation term contained in PTT, also acts as the source for sustaining a stationary power transport related to waveguide. Specifically, the input power is inputted into the section by passing through input port, and it is transformed into two parts by the section, where one part is finally outputted from the section by passing through output port and the other part is finally used to contribute the energy stored in the section.

On the input port, the tangential electric/magnetic field and the equivalent magnetic/electric current have the same magnitude and phase but perpendicular directions, so the IPO can be expressed in terms of several somewhat different forms, such as $\mathrm{EH}$, JE, and HM interaction forms. On the output port, the travelling-wave modes, which one-directionally propagate along the waveguide section from input port to output port, satisfy the travelling-wave condition, which can be viewed as a counterpart of the famous Sommerfeld's radiation condition for the radiative EM fields distributing at infinity. Employing the definitions of the equivalent currents on input port, the tangential field continuation conditions on waveguide wall, and the travelling-wave condition on output port, the dependent currents involved in the various forms of IPO can be effectively eliminated, and then the IPO with only independent currents is obtained.

Just like the antenna-related problem, PTT framework is also an effective framework for establishing waveguide-oriented modal analysis method - DMT. The PTT-DMT can construct a set of fundamental working modes for any pre-selected objective waveguide. The same as the classical eigen-modes constructed by EMT and the DMs constructed by antenna-oriented PTT-DMT (established by our group recently), the obtained fundamental modes are also energy-decoupled, which don't have net energy exchange in any integral period. In addition, the travelling-wave-type DMs satisfy the same dispersion equation as the one satisfied by the classical eigen-modes.

Compared with the classical EMT, the waveguide-oriented PTT-DMT has a very natural interface with the antenna-oriented PTT-DMT. This realizes an effective unification for the theoretical framework of waveguide-oriented and antenna-oriented modal analysis methods, and then leads to a possibility for doing modal analysis for whole waveguide-antenna combined system at once to be exhibited in our future studies.

\section{APPENDICES}

In these appendices, some detailed formulations related this paper are provided.

\section{A. On Travelling-Wave Conditions (8) and (9)}

The EM fields $(\boldsymbol{E}, \boldsymbol{H})$ on output port $S^{\mathrm{o}}$ satisfy the following tangential continuation conditions

$$
\boldsymbol{E}_{S_{-}^{0}}^{\mathrm{tan}}=\boldsymbol{E}_{S_{+}^{\mathrm{o}}}^{\mathrm{tan}} \text { and } \boldsymbol{H}_{S_{-}^{\mathrm{o}}}^{\mathrm{tan}}=\boldsymbol{H}_{S_{+}^{\mathrm{o}}}^{\mathrm{tan}}
$$

where $S_{-}^{0}$ and $S_{+}^{0}$ are, respectively, the left-side surface and right-side surface of $S^{\circ}$.

Based on surface equivalence principle, the fields $(\boldsymbol{E}, \boldsymbol{H})$ on $S_{-}^{o}$ can be expressed in terms of the functions of equivalent surface currents $\left(\boldsymbol{J}^{\mathrm{i}}, \boldsymbol{J}^{\mathrm{g}}, \boldsymbol{J}^{\mathrm{o}}, \boldsymbol{M}^{\mathrm{i}}, \boldsymbol{M}^{\mathrm{o}}\right)$ as follows:

$$
\begin{aligned}
& \boldsymbol{E}=\mathcal{E}\left(\boldsymbol{J}^{\mathrm{i}}+\boldsymbol{J}^{\mathrm{g}}-\boldsymbol{J}^{\mathrm{o}}, \boldsymbol{M}^{\mathrm{i}}-\boldsymbol{M}^{\mathrm{o}}\right) \text { on } S_{-}^{\mathrm{o}} \\
& \boldsymbol{H}=\mathcal{H}\left(\boldsymbol{J}^{\mathrm{i}}+\boldsymbol{J}^{\mathrm{g}}-\boldsymbol{J}^{\mathrm{o}}, \boldsymbol{M}^{\mathrm{i}}-\boldsymbol{M}^{\mathrm{o}}\right) \text { on } S_{-}^{\mathrm{o}}
\end{aligned}
$$

where the operators are the same as the ones defined after (3).

For the one-directionally propagating travelling-wave modes, their modal fields $(\boldsymbol{E}, \boldsymbol{H})$ on $S_{+}^{\circ}$ can be expressed in terms of the functions of equivalent surface currents $\left(\boldsymbol{J}^{\mathrm{o}}, \boldsymbol{M}^{\mathrm{o}}\right)$ as follows:

$$
\begin{aligned}
& \boldsymbol{E}=\mathcal{E}\left(\boldsymbol{J}^{\mathrm{o}}, \boldsymbol{M}^{\mathrm{o}}\right) \text { on } S_{+}^{\mathrm{o}} \\
& \boldsymbol{H}=\mathcal{H}\left(\boldsymbol{J}^{\mathrm{o}}, \boldsymbol{M}^{\mathrm{o}}\right) \text { on } S_{+}^{\mathrm{o}}
\end{aligned}
$$

because: for the one-directionally propagating travelling-wave modes, their wall currents on the right-hand side of $S^{0}$ will not contribute the fields distributing on $S_{+}^{\circ}$. A rigorous and detailed mathematical proof for (23) and (24) can be found in [55, Sec. 3.2.1], and some specific numerical verifications employing (23) and (24) are provided in the Secs. II-D, II-E, II-F and III-B of this paper.

Substituting (21)-(24) into (20), the travelling-wave conditions (8) and (9) are immediately obtained. In fact, the travelling-wave conditions are indispensable for establishing transformation (10). When the travelling-wave conditions are ignored, it cannot be guaranteed that the modes outputted from the modal decoupling equation are consistent with the classical eigen-modes.

\section{B. Integral Equations Used to Eliminate the Dependent Var- iables Contained in IPO (19)}

The currents involved in (18) are not independent, and they satisfy the following integral equations

$$
\begin{aligned}
& {\left[\mathcal{H}\left(\boldsymbol{J}^{\mathrm{i}}+\boldsymbol{J}^{\mathrm{f}}-\boldsymbol{J}^{\mathrm{o}}, \boldsymbol{M}^{\mathrm{i}}+\boldsymbol{M}^{\mathrm{f}}-\boldsymbol{M}^{\mathrm{o}}\right)\right]_{S_{+}^{\mathrm{i}}}^{\text {tan }}=\boldsymbol{J}^{\mathrm{i}} \times \boldsymbol{z}} \\
& {\left[\mathcal{E}\left(\boldsymbol{J}^{\mathrm{i}}+\boldsymbol{J}^{\mathrm{f}}-\boldsymbol{J}^{\mathrm{o}}, \boldsymbol{M}^{\mathrm{i}}+\boldsymbol{M}^{\mathrm{f}}-\boldsymbol{M}^{\mathrm{o}}\right)\right]_{S_{+}^{\mathrm{i}}}^{\mathrm{tan}}=\boldsymbol{z} \times \boldsymbol{M}^{\mathrm{i}}} \\
& {\left[\mathcal{E}\left(\boldsymbol{J}^{\mathrm{i}}+\boldsymbol{J}^{\mathrm{f}}-\boldsymbol{J}^{\mathrm{o}}, \boldsymbol{M}^{\mathrm{i}}+\boldsymbol{M}^{\mathrm{f}}-\boldsymbol{M}^{\mathrm{o}}\right)\right]_{S_{-}^{\mathrm{f}}}^{\text {tan }}=\left[\mathcal{E}_{0}\left(-\boldsymbol{J}^{\mathrm{f}},-\boldsymbol{M}^{\mathrm{f}}\right)\right]_{S_{+}^{\mathrm{f}}}^{\text {tan }}} \\
& {\left[\mathcal{H}\left(\boldsymbol{J}^{\mathrm{i}}+\boldsymbol{J}^{\mathrm{f}}-\boldsymbol{J}^{\mathrm{o}}, \boldsymbol{M}^{\mathrm{i}}+\boldsymbol{M}^{\mathrm{f}}-\boldsymbol{M}^{\mathrm{o}}\right)\right]_{S_{-}^{\mathrm{f}}}^{\text {tan }}=\left[\mathcal{H}_{0}\left(-\boldsymbol{J}^{\mathrm{f}},-\boldsymbol{M}^{\mathrm{f}}\right)\right]_{S_{+}^{\mathrm{f}}}^{\text {tan }}} \\
& {\left[\mathcal{E}\left(\boldsymbol{J}^{\mathrm{i}}+\boldsymbol{J}^{\mathrm{f}}-\boldsymbol{J}^{\mathrm{o}}, \boldsymbol{M}^{\mathrm{i}}+\boldsymbol{M}^{\mathrm{f}}-\boldsymbol{M}^{\mathrm{o}}\right)\right]_{S_{-}^{\mathrm{o}}}^{\text {tan }}=\left[\mathcal{E}\left(\boldsymbol{J}^{\mathrm{o}}, \boldsymbol{M}^{\mathrm{o}}\right)\right]_{S_{+}^{\mathrm{o}}}^{\text {tan }}} \\
& {\left[\mathcal{H}\left(\boldsymbol{J}^{\mathrm{i}}+\boldsymbol{J}^{\mathrm{f}}-\boldsymbol{J}^{\mathrm{o}}, \boldsymbol{M}^{\mathrm{i}}+\boldsymbol{M}^{\mathrm{f}}-\boldsymbol{M}^{\mathrm{o}}\right)\right]_{S_{-}^{\mathrm{o}}}^{\text {tan }}=\left[\mathcal{H}\left(\boldsymbol{J}^{\mathrm{o}}, \boldsymbol{M}^{\mathrm{o}}\right)\right]_{S_{+}^{\mathrm{o}}}^{\text {tan }}}
\end{aligned}
$$

Here, (25) and (26) are based on the definitions of $\boldsymbol{J}^{\mathrm{i}}$ and $\boldsymbol{M}^{\mathrm{i}}$ respectively; (27) and (28) are based on the tangential electric and magnetic field continuation conditions on $S^{\mathrm{f}}$ respectively; (29) and (30) are based on the travelling-wave condition on $S^{0}$. 


\section{REFERENCES}

[1] IEEE, The Authoritative Dictionary of IEEE Standards Terms, 7th. New York: IEEE Press, 2000.

[2] R. E. Collin, Field Theory of Guided Waves, 2nd. New York: IEEE Press, 1991.

[3] R. E. Collin, Foundations for Microwave Engineering. New York: IEEE Press, 2001.

[4] C. A. Balanis, Advanced Engineering Electromagnetics, 2nd. New York: Wiley, 2012.

[5] D. M. Pozar, Microwave Engineering. New York: Wiley, 2012.

[6] J. L. Volakis, Antenna Engineering Handbook. New York: McGraw-Hill, 2009.

[7] W. L. Stutzman and G. A. Thiele, Antenna Theory and Design, 3th. Hoboken: Wiley, 2013.

[8] C. A. Balanis, Antenna Theory: Analysis and Design, 4th. Hoboken: Wiley, 2016.

[9] W. C. Chew, Waves and Fields in Inhomogeneous Media. New York: IEEE Press, 1995.

[10] R.-B. Hwang, Periodic Structures: Mode-Matching Approach and Applications in Electromagnetic Engineering. Singapore: John Wiley \& Sons, Singapore, 2013.

[11] G. V. Eleftheriades, A. S. Omar, L. P. B. Katehi and G. M. Rebeiz, "Some important properties of waveguide junction generalized scattering matrices in the context of the mode matching technique," IEEE Trans. Microw. Theory Techn., vol. 42, no. 10, pp. 1896-1903, Oct. 1994.

[12] R. R. Collmann and F. M. Landstorfer, "Calculation of the field radiated by horn-antennas using the mode-matching method," IEEE Trans. Antennas Propag., vol. 43, no. 8, pp. 876-880, Aug. 1995.

[13] L. A. Francavilla, J. S. McLean, H. D. Foltz, and G. E. Crook, "Mode-matching analysis of top-hat monopole antennas loaded with radially layered dielectric," IEEE Trans. Antennas Propag., vol. 47, no. 1, pp. 179-185, Jan. 1999.

[14] H. Esteban, S. Cogollos, V. E. Boria, A. S. Blas and M. Ferrando, "A new hybrid mode-matching/numerical method for the analysis of arbitrarily shaped inductive obstacles and discontinuities in rectangular waveguides," IEEE Trans. Microw. Theory Techn., vol. 50, no. 4, pp. 1219-1224, Apr. 2002.

[15] S. P. Skobelev and P. S. Kildal, "Mode-matching modeling of a hard conical quasi-TEM horn realized by an EBG structure with strips and vias," IEEE Trans. Antennas Propag., vol. 53, no. 1, pp. 139-143, Jan. 2005.

[16] E. C. Titchmarsh, Eigenfunction Expansions Associated with Second-order Differential Equations, 2nd. London: Oxford University Press, 1962.

[17] A. Zettl, Sturm-Liouville Theory. American Mathematical Society, 2005.

[18] M. A. Al-Gwaiz, Sturm-Liouville Theory and Its Applications. Springer, 2008.

[19] F. V. Atkinson and A. B. Mingarelli, Multiparameter Eigenvalue Problems: Sturm-Liouville Theory. CRC Press, 2011.

[20] P. M. Morse and H. Feshbach, Methods of Theoretical Physics. New York: McGraw-Hill, 1953.

[21] R. Courant and D. Hilbert, Methods of Mathematical Physics. New York: Wiley, 1989.

[22] G. Gonzalez and V. R. Johnson, "Propagation in a rectangular waveguide partially filled with a linearly varying dielectric," IEEE Trans. Microw. Theory Techn., vol. 18, no. 7, pp. 404-406, Jul. 1970.

[23] K. F. Casey, "On Inhomogeneously filled rectangular waveguides," IEEE Trans. Microw. Theory Techn., vol. 21, no. 8, pp. 566-567, Aug. 1973.

[24] A. D. Berk, "Variational principles for electromagnetic resonators and waveguides," IEEE Trans. Antennas Propag., vol. 4, no. 2, pp. 104-111, Apr. 1956.

[25] R. E. Collin and R. M. Vaillancourt, "Application of Rayleigh-Ritz method to dielectric steps in waveguides," IEEE Trans. Microw. Theory Techn., vol. 5, no. 3, pp. 177-184, Mar. 1957.

[26] C. T. Liu and C. H. Chen, "A variational theory for wave propagation in inhomogeneous dielectric slab loaded waveguides," IEEE Trans. Microw. Theory Techn., vol. 29, no. 8, pp. 805-812, Aug. 1981.
[27] L. G. Chambers, "An approximate method for the calculation of propagation constants for inhomogeneously filled waveguides," Quart. J. Mech. And Appl. Math., vol. 7, pt. 3, pp. 299-316, Sep. 1954.

[28] D. A. Holmes, "Propagation in rectangular waveguide containing inhomogeneous, anisotropic dielectric," IEEE Trans. Microw. Theory Techn., vol. 12, no. 2, pp. 152-155, Mar. 1964.

[29] A. Wexler and D. A. Holmes, "Acceptable mode types for inhomogeneous media," IEEE Trans. Microw. Theory Techn., vol. 13, no. 6, pp. 875-878, Nov. 1965.

[30] B. M. Azizur and J. B. Davies, "Penalty function improvement of waveguide solution by finite elements," IEEE Trans. Microw. Theory Techn., vol. 32, no. 8, pp. 922-928, Aug. 1984.

[31] T. Angkaew, M. Matsuhara, and N. Kumagai, "Finite-element analysis of waveguide modes: A novel approach that eliminates spurious modes," IEEE Trans. Microw. Theory Techn., vol. 35, no. 2, pp. 117-123, Feb. 1987.

[32] M. Israel and R. Miniowitz, "Hermitian finite-element method for inhomogeneous waveguides," IEEE Trans. Microw. Theory Techn., vol. 38, no. 9,pp. 1319-1327, Sep. 1990.

[33] L. Nuño, J. V. Barbastre, and H. Castañé, "Analysis of general lossy inhomogeneous and anisotropic waveguides by the finite-element method (FEM) using edge elements," IEEE Trans. Microw. Theory Techn., vol. 45, no. 3, pp. 446-449, Mar. 1997.

[34] J. M. Jin, The Finite Element Method in Electromagnetics, 2nd. New York: John Wiley \& Sons. Inc., 2002.

[35] A. V. Taflove, Computational Electrodynamics: The Finite-Difference Time-Domain Method, 2nd. Artech House, 2000.

[36] S. F. Shu, P. M. Goggans and A. A. Kishk, "Computation of cutoff wavenumbers for partially filled waveguide of arbitrary cross section using surface integral formulations and the method of moments," IEEE Trans. Microw. Theory Techn., vol. 41, no. 6, pp. 1111-1118, Jun. 1993.

[37] Q.-H. Liu and W. C. Chew, "Analysis of discontinuities in planar dielectric waveguides: An eigenmode propagation method," IEEE Trans. Microw. Theory Techn., vol. 39, no. 3, pp. 422-430, Mar. 1991.

[38] C. M. Herzinger, C. C. lu, Ta A. DeTemle and W. C. Chew, "The semiconductor waveguide facet reflectivity problem," IEEE J. Quantum Elec., Vol. 29, no. 8, pp. 2273-2281, Aug. 1993.

[39] L. J. Chu, "Physical limitations of omni-directional antenna," J. Appl. Phys., vol. 19, pp. 1163-1175, 1948.

[40] S. M. Mikki and Y. M. M. Antar, "A theory of antenna electromagnetic near field - Part I," IEEE Trans. Antennas Propag., vol. 59, no. 12, pp. 4691-4705, Dec. 2011.

[41] S. M. Mikki and Y. M. M. Antar, "A theory of antenna electromagnetic near field - Part II," IEEE Trans. Antennas Propag., vol. 59, no. 12, pp. 4706-4724, Dec. 2011.

[42] K. F. Lee and W. Chen, Advances in Microstrip and Printed Antennas. New York: Wiley, 1997.

[43] K.-M. Luk and K.-W. Leung, Dielectric Resonator Antennas. Hertfordshire U.K.: Research Studies Press, 2003.

[44] R. J. Garbacz, "Modal expansions for resonance scattering phenomena," Proc. IEEE, vol. 53, no. 8, pp. 856-864, Aug. 1965.

[45] R. J. Garbacz, "A generalized expansion for radiated and scattered fields," Ph.D. dissertation, Dept. Elect. Eng., The Ohio State Univ., Columbus, OH, USA, 1968.

[46] R. J. Garbacz and R. H. Turpin, "A generalized expansion for radiated and scattered fields," IEEE Trans. Antennas Propag., vol. AP-19, no. 3, pp. 348-358, May 1971.

[47] R. F. Harrington and J. R. Mautz, Theory and Computation of Characteristic Modes for Conducting Bodies. Interaction Notes, Note 195, Syracuse Univ., Syracuse, New York, USA, Dec. 1970.

[48] R. F. Harrington and J. R. Mautz, "Theory of characteristic modes for conducting bodies," IEEE Trans. Antennas Propag., vol. AP-19, no. 5, pp. 622-628, Sep. 1971.

[49] R. F. Harrington, J. R. Mautz, and Y. Chang, "Characteristic modes for dielectric and magnetic bodies," IEEE Trans. Antennas Propag., vol. AP-20, no. 2, pp. 194-198, Mar. 1972.

[50] Y. Chang and R. F. Harrington, "A surface formulation for characteristic modes of material bodies," IEEE Trans. Antennas Propag., vol. 25, no. 6, pp. 789-795, Nov. 1977.

[51] M. Cabedo-Fabres, E. Antonino-Daviu, and A. Valero-Nogueira, et al., "The theory of characteristic modes revisited: a contribution to the design of antennas for modern applications," IEEE Antennas Propag. Mag., vol. 49 , no. 5, pp. 52-68, Oct. 2007. 
[52] M. Vogel, G. Gampala, and D. Ludick, et al., "Characteristic mode analysis: putting physics back into simulation," IEEE Antennas Propag. Mag., vol. 57, no. 2, pp. 307-317, Apr. 2015.

[53] Y. K. Chen and C.-F. Wang, Characteristic Modes: Theory and Applications in Antenna Engineering. Hoboken: Wiley, 2015.

[54] R. Z. Lian and M. Y. Xia, "Power transport theorem based decoupling mode theory for wave-port-fed transmitting antennas," with manuscript ID [AP2104-0777] submitted to IEEE Trans. Antennas Propag. on 14-Apr-2021, and being Under Review.

[55] R. Z. Lian, "Research on the power transport theorem based decoupling mode theory for transceiving systems," Post-Doctoral Reseach Report, Department of Electronics, School of Electronics Engineering and Computer Science, Peking University (PKU), Beijing, China, February 11, 2021. [Online]. Available: https://arxiv.org/abs/2103.01853.

Ren-Zun Lian received the B.S. degree in optical engineering from the University of Electronic Science and Technology of China (UESTC), Chengdu, China, in 2011, and received the $\mathrm{Ph} . \mathrm{D}$. degree in electromagnetic field and microwave technology from UESTC, Chengdu, China, in 2019.

$\mathrm{He}$ is currently a Post-Doctoral Research Fellow in Peking University (PKU), Beijing, China. His current research interests include mathematical physics, electromagnetic theory \& computation, microwave circuits \& components, and antenna theory \& design.

Ming-Yao Xia (M'00-SM'03) received the Master and Ph. D degrees in electrical engineering from the Institute of Electronics, Chinese Academy of Sciences (IECAS), in 1988 and 1999, respectively. From 1988 to 2002, he was with IECAS as an Engineer and a Senior Engineer. He was a Visiting Scholar at the University of Oxford, U.K., from October 1995 to October 1996. From June 1999 to August 2000 and from January 2002 to June 2002, he was a Senior Research Assistant and a Research Fellow, respectively, with the City University of Hong Kong. He joined Peking University as an Associate Professor in 2002 and was promoted to Full Professor in 2004. He moved to the University of Electronic Science and Technology of China as a Chang-Jiang Professor nominated by the Ministry of Education of China in 2010. He returned to Peking University after finishing the appointment in 2013. He was a recipient of the Young Scientist Award of the URSI in 1993. He was awarded the first-class prize on Natural Science by the Chinese Academy of Sciences in 2001. He was the recipient of the Foundation for Outstanding Young Investigators presented by the National Natural Science Foundation of China in 2008. He served as an Associate Editor for the IEEE Transactions on Antennas and Propagation. His research interests include electromagnetic theory, numerical methods and applications, such as wave propagation and scattering, electromagnetic imaging and probing, microwave remote sensing, antennas and microwave components. 